\title{
Simulation of an Intracellular Differential Equation Model of the Dynamics of Malaria with Immune Control and Treatment
}

\author{
Titus Ifeanyi Chinebu, Edmund Onwubiko Ezennorom, John U Okwor \\ Department of Computer Science, Madonna University, Elele, Okija, Nigeria
}

\begin{abstract}
We designed a simulation of an intracellular differential equation model of the dynamics of malaria with immune control and treatment which considered malaria parasites in the liver and blood. We considered transmission dynamics of malaria and the interaction between the infection in the liver and blood. The disease free equilibrium of our model was asymptotically stable when the basic reproduction number is less than one and unstable when it is greater than one. Numerical simulations show that if the immune response is strong with effective treatment, malaria infection will be cleared from an infectious human host. A treatment strategy using highly effective drugs against malaria parasites with strong immune response can reduce malaria progression and control the disease.
\end{abstract}

Keyword: Mathematical model, Malaria parasite, Hepatocyte, Erythrocyte, Meroziote, Sporozoite, Immune response, Treatment.

\section{INTRODUCTION}

Malaria is a life threatening mosquito borne blood disease caused by a plasmodium parasite and children are particularly susceptible to the disease. In 2015, an estimated 306,000 children under 5 years of age were kills mostly in the African region (WHO World Malaria Report, 2015). Once transmitted to the human by a blood feeding Anopheles mosquito, the parasites initially multiply in the human liver, before they progress to the pathologic blood stage. Immediately the parasite (sporozoites) first enters the human host, there is a pre- erythrocytic development. After inoculation into a human by female anopheles mosquito, sporozoites invade hepatocytes in the host liver and multiply there for $5-12$ days, forming hepatic schizonts. These then burst, liberating merozoites into the bloodstream where they subsequently invade red blood cells. These blood infections can last for months, and only once sexual precursor cells, the gametocytes have matured, the malaria parasite are able to leave the human host and to continue the life cycle in the insect vector. In the mosquito midgut, the parasite are able to differentiate into their sexual forms, the female macrogametes and male microgametes, and to then undergo sexual reproduction in order to newly combine their chromosomal sets. The midgut phase lasts for approximately 20 hours and includes two phases of stage conversion, the rapid conversion gametocyte into fertile gametes upon activation and the conversion of zygotes into motile and invasive ookinates that once formed, immediately exit the gut lumen by traversing the midgut epithelial cell layer. Subsequently, the ookinates settle down at the basal site of the midgut epithelium and convert to sessil oocysts in which sporogonic replication takes place. This replication phase requires roughly 2 weeks and results in the formation of infective sporozoites that migrate to the salivary glands to be released into the human dermis with the next bit of the mosquito wherewith the life cycle of plasmodium is completed (Aly et al, 2009; Ghosh and Jacobs-Lorena, 2009; Kuehn and Pradel, 2010; Menard et al, 2013; Bennink et al, 2016).

Sexual precursor cells the intraerythrocytic gametocytes develop in the human blood in response to the stress factor (Pradel, 2007;Kuehn and Pradel, 2010). A time period of about 10 days is required for gametocyte development in P. falciparum, during which they pass five morphological stages. Once the gametocytes mature and is ingested with the blood meal of an Anepheles mosquito, they are activated in the mosquito midgut by environmental stimuli, and gametogenesis is initiated. Signals inducing gamete 
formation include a drop of temperature by approximately $5^{0} \mathrm{C}$ which is mandatory for gametocyte activation and the presence of the mosquito derived molecule Xanthurenic acid (XA), a metabolic intermediate of the tryptophan catabolism. An additional trigger of game to genesis is the increase of extracellular $\mathrm{pH}$ from 7.2 to about 8 (Kawamoto et al, 1991;Billker et al, 1997; Garcia et al, 1998; Sologub et al, 2011).

The periodic bouts of fever that occur in the malaria coincide with the synchronized rupture of plasmodium-infected red blood cells. This causes the release of parasites en masses into the blood stream, along with pigments and toxins that have accumulated inside the red blood cells as a result of the parasites metabolic activities. The presence of large quantities of parasite material in the blood triggers a dramatic immune response, mediated by the secretion of cytokine modules by the cells of the immune system (Hommel and Gilles, 1998). Some cytokines such as tumor necrosis factor (TNF), interferon gamma, interleukin 12 and interleukin 18 enhances the immune response, stimulating macrophages and other immune cells to destroy parasites by phagoytosis and by the production of toxins. Other cytokines include interleukin 4, interleukin 10 and TGF-beta help to regulate the immune response by dampening these effects (Malaguarnera and Musumeci, 2002).

\section{Related Literatures}

Chi-Johnston (2012) develops and analyze a comprehensive simulation model of $\mathrm{P}$. falciparum within-host malaria infection and transmission in immunologically-naïve humans. There model incorporates the entire lifecycle of P. falciparum starting with the asexual blood stage forms responsible for disease, the onset of symptoms, the development and maturation of sexual stage forms (gametocytes) that are transmissible to Anopheles mosquitoes, and human to mosquito infectivity. The model components were parameterized from malaria therapy data and from arrange of other studies to simulate individual infections such that the ensemble is statistically consistent with the full range of patient responses to infection. Human infectivity was modeled over the course of untreated infections and the effects were examined in relation to transmission intensity expressed in terms of the basic reproduction number. Adamu (2014) developed a mathematical model to study the dynamics of malaria disease in a population and consideration were given to the interaction between the parasites and the host (human beings), such that the susceptible and the infected classes were allowed to interact freely without quarantining any of the either classes. In their model, first order equation that describes the dynamics of the susceptible class and the infected class under the influence of the parasite was used. The result of the qualitative and stability analysis showed that if preventive measure is not put in place, the susceptible and infected classes will reach a stable equilibrium point which can be disastrous to the population and they recommended specific measures of controlling the disease.

Johansson and Leander (2010) used three compartment of susceptible, infectious and recovered in their work and they showed that the recovered are neither quarantined nor removed from the entire population rather they enter the susceptible class again. Tabo et al (2017) developed a mathematical model which considers the dynamics of $\mathrm{P}$. falciparum malaria from the liver to the blood in the human host and then to the mosquito. There results indicated that the infection rate of merozoites, the rate of sexual reproduction in gametocytes, burst size of both hepatocytes and erythrocytes are more sensitive parameters for the onset of the disease. They suggested that a treatment strategy using highly effective drugs against such parameters can reduce on malaria progression and control the disease. There numerical simulation shows that drugs with efficacy above $90 \%$ boost healthy cells and clear parasites in human host. However, all these models are limited to treatment, non considered treatment and immune response. Here, we formulated a more detailed model to study the intracellular dynamics of malaria with immune control and treatment using mathematical model. Our aim is to study the interaction between malaria and immune response with treatment measure through a mathematical model and carry out a sensitivity analysis to determine the parameters that controls the disease.

\section{MODEL FORMULATION}

\subsection{THE ASSUMPTIONS OF THE MODEL}

The disease is spread by transmission through mosquito to human interaction;

By immunology memory, the immunity of infected /infectious individuals might be rapidly restored when they are re exposed to the infection. 
Individual can loss their immunity when they are not susceptible.

continuously exposed to the parasite and go back to Treatment can either be successful or fail.

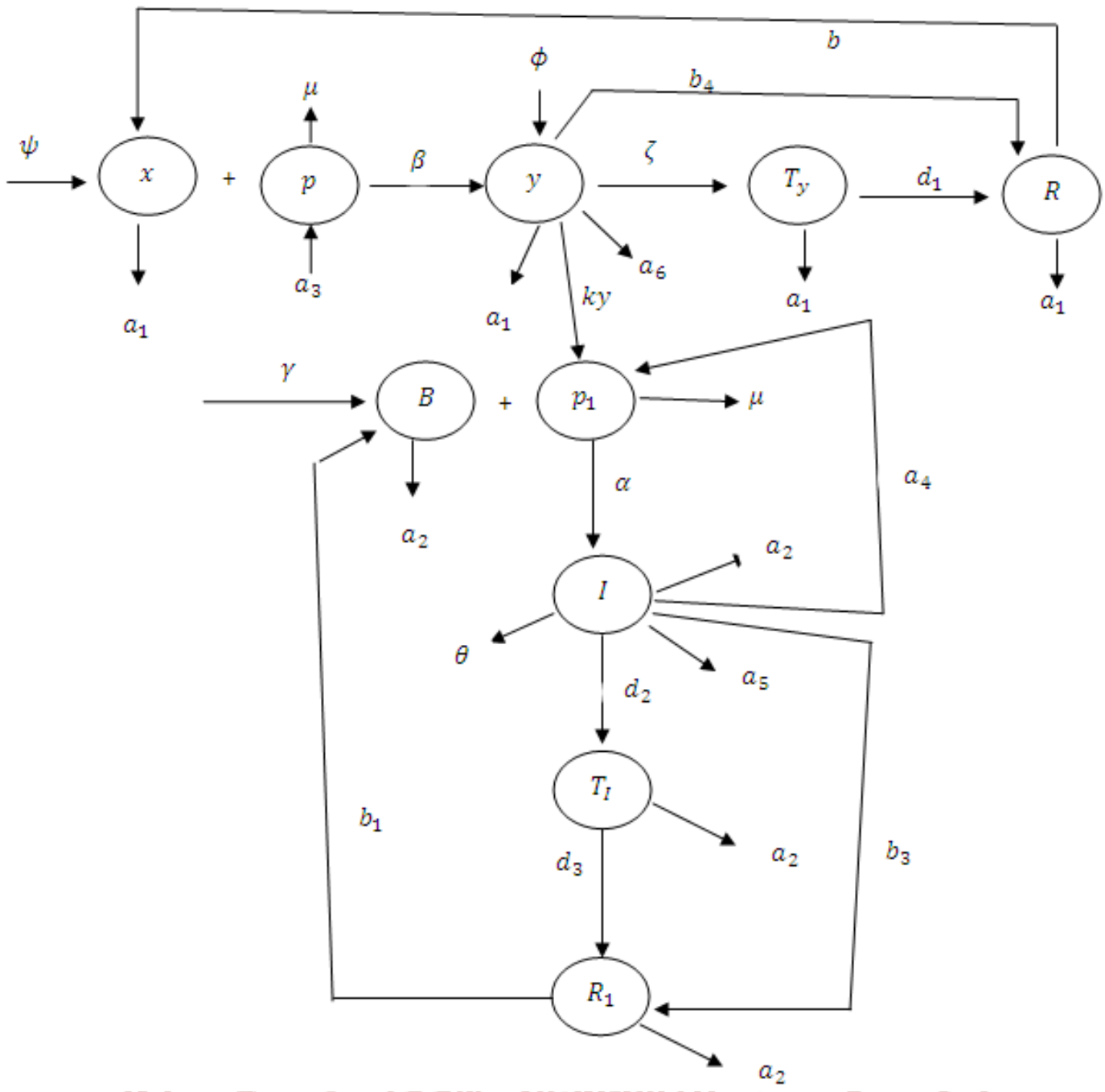

Fig 1: Flow diagram of malaria treatment model

\subsection{Variables (Compartments)}

The model is made up of ten (10) compartments which comprises of $(x)$, Uninfected hepatocytes (liver cells), $(p)$,Free sporozoites (malaria parasites in the liver), $(y)$, Infected hepatocytes, $\left(T_{y}\right)$, Treated infected hepatocytes, $(R)$, Recovered hepatocytes, $\left(p_{1}\right)$, Free merozites (malaria parasite in the blood), $(B)$, Uninfected erythrocytes (red blood cell), $(I), \gamma$ Infecfed erythrocytes, $\left(T_{I}\right)$, Treated infected erythrocytes and $\left(R_{1}\right)$, Recovered erythrocytes.

\section{Parameters}

$\psi$ recruitment level of uninfectec hepatocytes

$a_{1}$ natural death rate of both uninfected, infected, $a_{5}$ and recovered hepatocytes

$\beta \quad$ rate at which hepatocytes are being infected

$\mu \quad$ death rate of malaria parasites (sporozoites) $a_{3}$ rate at which free sporozoite is inoculated into the hepatocyte by mosquitoes treatment rate of infected hepatocytes movement rate of treated hepatocytes to recovered class

natural death rate of erythrocytes (red blood cells)

recruitment level of erythrocytes from bone marrow

$\alpha \quad$ rate at which the uninfected erythrocytes are being infected

$a_{4} \quad$ rate at which the infected erythrocytes produce free parasites (merozoite)

disease induced death rate of infected erythrocytes

$a_{6}$ disease induced death rate of infected hepatocytes

$\phi \quad$ the rate at which infected hepatoctesproliferate 
International Journal of Trend in Scientific Research and Development (IJTSRD) ISSN: 2456-6470

$\theta$ the rate at which infected erythrocyte proliferate

$d_{2} \quad$ rate treatment of the infected erythrocytes

$d_{3} \quad$ movement level of infected erythrocytes to the recovered class

ky rate at which infected hepatocyte produces meroziotes

$b_{3}$ recovered red blood cells due to immune response

$b_{4} \quad$ recovered liver cells due to immune response

$b$ movement rate of the recovered hepatocytes to susceptible class.

$b_{1} \quad$ movement rate of the recovered red blood cells to susceptible class

\subsection{The model equation}

$$
\begin{gathered}
\frac{d x}{d t}=\psi-a_{1} x-\beta x p+b R \\
\frac{d p}{d t}=a_{3} y-\mu p \\
\frac{d y}{d t}=\beta x p+\phi y-\zeta y-a_{1} y-a_{6} y-k y p_{1}-b_{4} y \\
\frac{d T_{y}}{d t}=\zeta y-a_{1} T_{y}-d_{1} T_{y} \text { ternatio }
\end{gathered}
$$$$
\frac{d R}{d t}=d_{1} T_{y}-a_{1} R-b R
$$$$
+b_{4} y
$$$$
\frac{d B}{d t}=\gamma-\alpha B p_{1}-a_{2} B+b_{1} R_{1}
$$$$
\frac{d p_{1}}{d t}=a_{4} I-\mu p_{1}
$$$$
\frac{d I}{d t}=\alpha B p_{1}+\theta I-d_{2} I-a_{2} I-a_{5} I-b_{3} I
$$$$
\frac{d T_{I}}{d t}=d_{2} I-d_{3} T_{I}-a_{2} T_{I}
$$$$
\frac{d R_{1}}{d t}=d_{3} T_{I}-a_{2} R_{1}-b_{1} R_{1}+b_{3} I
$$

$$
\begin{aligned}
& \text { Let the initial conditions be } \\
& \qquad \begin{aligned}
x(0)=x_{0}, y(0) & =y_{0}, T_{y}(0)=T_{y 0}, R(0)=R_{0}, B(0) \\
& =B_{0}, I(0)=I_{0}, T_{I}(0)=T_{I 0}, R_{1}(0) \\
& =R_{10} \quad(3.2)
\end{aligned}
\end{aligned}
$$

\subsection{Equilibrium state analysis}

The equilibrium state is the uninfected state and for malaria infection to manifest, the individual must be bitten by an infected mosquito. Also, the rate of change in sporozoites and merozoites concentration will be positively much faster than that of the cell concentration and for it to clear, the rate of change in sporozoites and merozoites concentration will be negatively much faster than that of the cell concentration.

Notice from equation (3.1) that the production rate of the parasite $(p)$, from the livercells is proportional to the rate at which they are removed and are at equilibrium, i.e., $a_{3} y-\mu p=0$. So we let

$$
p=\frac{a_{3} y}{\mu}
$$

Also from equation (3.1), we observe that the rate of production of the parasite $\left(p_{1}\right)$, from the red blood cells is proportional to the rate at which the are removed and are at equilibrium, i.e., $a_{4} I-\mu p_{1}=0$. So we let

$$
p_{1}=\frac{a_{4} I}{\mu}
$$

Substituting $p=\frac{a_{3} y}{\mu}$ and $p_{1}=\frac{a_{4} I}{\mu}$ into equation (3.1) reduces the model to eight non linear ordinary differential equations and this will make the quantitative analysis much easier. Now we rewrite the equations as:

$$
\frac{d x}{d t}=\psi-a_{1} x-\beta x \frac{a_{3} y}{\mu}+b R
$$

$$
\begin{aligned}
& \frac{d y}{d t}=\beta x \frac{a_{3} y}{\mu}+\phi y-\zeta y-a_{1} y-a_{6} y-k y \frac{a_{4} I}{\mu} \\
& \text { and } \quad \frac{d T_{y}}{d t}=\zeta y-b_{4} T_{y}-d_{1} T_{y} \\
& \frac{d R}{d t}=d_{1} T_{y}-a_{1} R-b R \\
& \qquad \frac{d B}{d t}=\gamma-\alpha B \frac{a_{4} I}{\mu}-a_{2} B+b_{1} R_{1} \\
& \frac{d I}{d t}=\alpha B \frac{a_{4} I}{\mu}+\theta I-d_{2} I-a_{2} I-a_{5} I-b_{3} I \\
& \frac{d T_{I}}{d t}=d_{2} I-d_{3} T_{I}-a_{2} T_{\mathrm{I}} \\
& \frac{d R_{1}}{d t}=d_{3} T_{I}-a_{2} R_{1}-b_{1} R_{1}+b_{3} I
\end{aligned}
$$

Because the model s are items of populations and in two interacting cell population, that is, the liver cells which produces sporozoites and the red blood cells which produces merozoites. The liver cell and the red blood cell population size at time $t$ are respectively represented as

$$
\begin{aligned}
x(t)+y(t)+ & T_{y}(t)+R(t) \\
& =N(t) \operatorname{and} B(t)+I(t)+T_{I}(t) \\
& +R_{1}(t)=N_{1}(t)
\end{aligned}
$$




\subsection{Existence and Positivity of solutions}

Having that all the parameters in equation (3.1) are non negative, we assume a stable population with per capita recruitment of susceptible liver cells, susceptible red blood cells, death rate of liver cells both natural and disease induced, death rate of red blood cells both natural and disease induced. At this point we normalize the population size of both the liver cells and red blood cells to one (1) each and show that the system is epidemiologically and mathematically well-posed in the feasible region $\Gamma$ given by

where

$$
\Gamma=A_{L} \times A_{r} \subset \mathbb{R}_{+}^{3} \times \mathbb{R}_{+}^{3}
$$

$$
\begin{aligned}
A_{L}=\left\{\left(x, y, T_{y}\right)\right. & \left.\in \mathbb{R}_{+}^{3}: N \leq \frac{\psi}{a_{1}}\right\} \text { and } A_{r} \\
= & \left\{\left(B, I, T_{I}\right) \in \mathbb{R}_{+}^{3}: N_{1} \leq \frac{\gamma}{a_{2}}\right\}
\end{aligned}
$$

At this point we let the time derivative of $A_{L}(t)$ and $A_{r}(t)$ along solutions of system (3.2) for liver cells and red blood cells respectively be calculated thus,

$$
\begin{aligned}
\mathrm{A}_{\mathrm{L}}(\mathrm{t})=x(t)+ & y(t) \\
& +T_{y}(t) \\
\mathrm{A}_{\mathrm{L}}(\mathrm{t})=\psi- & a_{1} x-\beta x \frac{a_{3} y}{\mu}+b\left(1-B-I-T_{I}\right) \\
& +\beta x \frac{a_{3} y}{\mu} \\
+\phi y-\zeta y- & a_{1} y-a_{6} y-k y \frac{a_{4} I}{\mu}+\zeta y-a_{1} T_{y} \\
- & d_{1} T_{y}-b_{4} y
\end{aligned}
$$

where

$$
A_{L}=x+y+T_{y}
$$

Remember that in the absence of the diseased $d_{1} T_{y}, k y \frac{a_{4} I}{\mu}, \phi y, b_{4} y^{2}$ and $a_{6} y$ will be equal to

Theorem 1: There exists a domain $\Gamma$ in which the solution set $\left\{x, y, T_{y}, B, I, T_{I}\right\}$ is contained and bounded.

Proof: Given the solution set $\left\{x, y, T_{y}, B, I, T_{I}\right\}$ with positive initial conditions (3.2), we let the liver population be represented as

$$
x+y+T_{y}+R=1
$$

$$
\Rightarrow R=1-x-y-T_{y}
$$

while the red blood cell population is represented as

$$
\begin{aligned}
& B+I+T_{I}+R_{1} \\
& =1 \quad \Rightarrow R_{1}=1-B-I-T_{I}
\end{aligned}
$$

Omitting the equation for $R a n d R_{1}$ in our analysis gives equation (3) as

$$
\begin{aligned}
& \frac{d x}{d t}=\psi-a_{1} x-\beta x \frac{a_{3} y}{\mu}+b\left(1-B-I-T_{I}\right) \\
& \frac{d y}{d t}=\beta x \frac{a_{3} y}{\mu}+\phi y-\zeta y-a_{1} y-a_{6} y-k y \frac{a_{4} I}{\mu} \\
& -b_{4} y \\
& \frac{d T_{y}}{d t} \\
& =\zeta y-a_{1} T_{y} \\
& -d_{1} T_{y} \\
& \frac{d B}{d t}=\gamma-\alpha B \frac{a_{4} I}{\mu}-a_{2} B+b_{1}\left(1-B-I-T_{I}\right) \\
& \frac{d I}{d t}=\alpha B \frac{a_{4} I}{\mu}+\theta I-d_{2} I-a_{2} I-a_{5} I-b_{3} I \\
& \frac{d T_{I}}{d t}=d_{2} I-d_{3} T_{I}-a_{2} T_{I}
\end{aligned}
$$

zero. Then we obtain

$$
\begin{gathered}
\mathrm{A}_{\mathrm{L}}(\mathrm{t})=\psi-a_{1} x-a_{1} y-a_{1} T_{y}+b\left(1-\mathrm{A}_{\mathrm{L}}\right) \\
\mathrm{A}_{\mathrm{L}}(\mathrm{t})=\psi-a_{1}\left(x+y+T_{y}\right)+b\left(1-\mathrm{A}_{\mathrm{L}}\right) \\
\mathrm{A}_{\mathrm{L}}(\mathrm{t})=\psi-a_{1} \mathrm{~A}_{\mathrm{L}}+b-b \mathrm{~A}_{\mathrm{L}}
\end{gathered}
$$

$\mathrm{A}_{\mathrm{L}}(\mathrm{t})+\left(a_{1}+b\right) \mathrm{A}_{\mathrm{L}}$

$$
\begin{aligned}
& \leq \psi \\
& +b
\end{aligned}
$$

We shall integrate both sides of equation (3.8) using integrating factor method according to (Kar and Jana, 2013; Birkhoff and Roffa, 1989) to obtain:

$$
\begin{gathered}
A_{L}^{\prime}+P(t) d t=F(t) \\
A_{L} \leq e^{-\int P(t) d t}\left(\int e^{\int P(t) d t} F(t) d t+C\right)
\end{gathered}
$$

where $P(t)=a_{1}+b$ and $F(t)=\psi+b$. Let the integrating factor be

$$
r(t)=e^{\int P(t) d t}=e^{\int\left(a_{1}+b\right) d t}=e^{\left(a_{1}+b\right) t}
$$

Then integrating equation (3.8) by inputting $r(t)=$ $e^{\left(a_{1}+b\right) t}$ gives

$$
\begin{gathered}
A_{L}(t) \leq \frac{1}{r(t)}\left(\int r(t) \cdot F(t) d t+C\right) \\
\Rightarrow A_{L}(t) \leq \frac{1}{e^{\left(a_{1}+b\right) t}}\left(\int e^{\left(a_{1}+b\right) t} \cdot(\psi+b) d t+C\right) \\
A_{L}(t) \leq \frac{1}{e^{\left(a_{1}+b\right) t}}\left((\psi+b) \int e^{\left(a_{1}+b\right) t} d t+C\right) \\
A_{L}(t) \leq \frac{1}{e^{\left(a_{1}+b\right) t}}\left(\frac{(\psi+b)}{\left(a_{1}+b\right)} e^{\left(a_{1}+b\right) t}+C\right)
\end{gathered}
$$


International Journal of Trend in Scientific Research and Development (IJTSRD) ISSN: 2456-6470

$$
\begin{aligned}
& A_{L}(t) \\
& \leq \frac{(\psi+b)}{\left(a_{1}+b\right)} \\
& +C e^{-\left(a_{1}+b\right) t}
\end{aligned}
$$

Here, $\mathrm{C}$ is the constant of integration and if we let $t \rightarrow$ $\infty$ we have that

But

$$
A_{L}(t)=\frac{(\psi+b)}{\left(a_{1}+b\right)}=x+y+T_{y}
$$

$$
x \leq \frac{\psi}{a_{1}}
$$

Also,

$A_{r}(t)$

$$
=\mathrm{B}(\mathrm{t})+\mathrm{I}(\mathrm{t})+\mathrm{T}_{\mathrm{I}}(\mathrm{t})
$$

$$
\begin{aligned}
\mathrm{A}_{\mathrm{r}}(\mathrm{t})=\gamma-\alpha B & \frac{a_{4} I}{\mu}-a_{2} B+b_{1}\left(1-B-I-T_{I}\right) \\
+ & \alpha B \frac{a_{4} I}{\mu}
\end{aligned}
$$$$
+\theta I-d_{2} I-a_{2} I-a_{5} I-b_{3} I+d_{2} I-d_{3} T_{I}-a_{2} T_{I}
$$

is positively invariant and the systems for the liver cells and red blood cells are respectively well-posed epidemically and mathematically. Then for the initial starting point $A_{L} \in \mathbb{R}_{+}^{3}$ and $A_{r} \in \mathbb{R}_{+}^{3}$ the trajectory lies on $\Gamma$. Thus, we focus our attention only on the region $\Gamma$.

\subsection{Disease Free Equilibrium point}

To study the equilibrium state and analyze the stability of the system, we set the right side of equation (3.3) to zero. Thus, we have

$$
\begin{gathered}
\psi-a_{1} x-\beta x \frac{a_{3} y}{\mu}+b R=0 \\
\beta x \frac{a_{3} y}{\mu}+\phi y-\zeta y-a_{1} y-a_{6} y-k y \frac{a_{4} I}{\mu}-b_{4} y \\
=0 \\
\zeta y-a_{1} T_{y}-d_{1} T_{y}=0 \\
d_{1} T_{y}-a_{1} R-b R+b_{4} y \\
=0 \\
\gamma-\alpha B \frac{a_{4} I}{\mu}-a_{2} B+b_{1} R_{1}=0 \\
\alpha B \frac{a_{4} I}{\mu}+\theta I-d_{2} I-a_{2} I-a_{5} I-b_{3} I=0 \\
J O U d_{2} I-d_{3} T_{I}-a_{2} T_{I}=0
\end{gathered}
$$

$$
A_{r}=B+I+T_{I}
$$

Also, in the absence of the Tisease,

$\theta I, a_{5} I, b_{3} \operatorname{Iand}_{3} T_{I}$ will be zero. Then we have

$$
\begin{aligned}
& \mathrm{A}_{\mathrm{r}}(\mathrm{t})=\gamma-a_{2} B-a_{2} I-a_{2} T_{I}+b_{1}\left(1-\mathrm{A}_{\mathrm{r}}\right) \\
& \mathrm{A}_{\mathrm{r}}(\mathrm{t})=\gamma-a_{2}\left(B+I+T_{I}\right)+b_{1}\left(1-\mathrm{A}_{\mathrm{r}}\right) \\
& \quad \mathrm{A}_{\mathrm{r}}(\mathrm{t})=\gamma-a_{2} \mathrm{~A}_{\mathrm{L}}+b_{1}-b_{1} \mathrm{~A}_{\mathrm{L}} \\
& \mathrm{A}_{\mathrm{r}}(\mathrm{t})+\left(a_{2}+b_{1}\right) \mathrm{A}_{\mathrm{r}} \\
& \leq \gamma+b_{1}
\end{aligned}
$$

Using integrating factor method on equation (3.12), we have

$$
\begin{aligned}
& A_{r}(t) \\
& \leq \frac{\left(\gamma+b_{1}\right)}{\left(a_{2}+b_{1}\right)} \\
& +C_{1} e^{-\left(a_{2}+b_{1}\right) t}
\end{aligned}
$$

Here, C

is the constant of integration and if we let $t \rightarrow \infty$ we have that

But

$$
A_{r}(t)=\frac{\left(\gamma+b_{1}\right)}{\left(a_{1}+b_{1}\right)}=B+I+T_{I}
$$

$$
B \leq \frac{\gamma}{a_{2}}
$$

Observe from the dynamics describe by the systems (3.2), (3.10) and (3.14) that the region

$$
\Gamma=\left\{\left(x, \mathrm{y}, \mathrm{T}_{\mathrm{y}}, \mathrm{B}, \mathrm{I}, \mathrm{T}_{\mathrm{I}}\right) \in \mathbb{R}_{+}^{6}: \mathrm{N} \leq \frac{\psi}{\mathrm{a}_{1}}: \mathrm{N}_{1} \leq \frac{\gamma}{\mathrm{a}_{2}}\right\}
$$

If we label equation (3.15) as (3.15i) to (3.15viii), then (3.15ii) gives

$$
\begin{gathered}
\beta x \frac{a_{3} y}{\mu}+\phi y-\zeta y-a_{1} y-a_{6} y-k y \frac{a_{4} I}{\mu}-b_{4} y=0 \\
\left(\beta x \frac{a_{3}}{\mu}+\phi-\zeta-a_{1}-a_{6}-k \frac{a_{4} I}{\mu}-b_{4}\right) y=0
\end{gathered}
$$

From (3.15iii) we have

$$
\Rightarrow y=0
$$

$$
\zeta y-a_{1} T_{y}-d_{1} T_{y}=0
$$

But $y=0$, then we have

From (3.15iv) we obtain

$$
d_{1} T_{y}-a_{1} R-b R+b_{4} R=0
$$

Since $T_{y}=y=0$, we have

$$
\left(a_{1}+b\right) R=0 \Rightarrow R=0
$$

From (3.15i) we have

$$
\psi-a_{1} x-\beta x \frac{a_{3} y}{\mu}+b R=0
$$

But $y$ and $R$ are all equal to zero, then we have

$$
\psi-a_{1} x=0 \Rightarrow x=\frac{\psi}{a_{1}}
$$

From (3.15i) we get

$$
\alpha B \frac{a_{4} I}{\mu}+\theta I-d_{2} I-a_{2} I-a_{5} I-b_{3} I=0
$$


International Journal of Trend in Scientific Research and Development (IJTSRD) ISSN: 2456-6470

$$
\begin{gathered}
\left(\alpha B \frac{a_{4}}{\mu}+\theta-d_{2}-a_{2}-a_{5}-b_{3}\right) I=0 \\
\Rightarrow I=0
\end{gathered}
$$

From (3.15vii) we have

$$
d_{2} I-d_{3} T_{I}-a_{2} T_{I}=0
$$

But $I=0$, then

$$
\left(d_{3}+a_{2}\right) T_{\mathrm{I}}=0 \Rightarrow T_{\mathrm{I}}=0
$$

Substituting $T_{\mathrm{I}}=I=0 \mathrm{in}(3.15 \mathrm{viii})$ we obtain

$$
\left(a_{2}+b_{1}\right) R_{1}=0 \Rightarrow R_{1}=0
$$

Also, if we substitute $I=R_{1}=0$ into $(3.15 \mathrm{v})$ we get

$$
\gamma-a_{2} B=0 \Rightarrow B=\frac{\gamma}{a_{2}}
$$

There, the disease free equilibrium point of the model is given as

$$
\begin{aligned}
& \Phi=\left(x, y, T_{y}, R, B, I, T_{I}, R_{1}\right) \\
& =\left(\frac{\psi}{a_{1}}, 0,0,0, \frac{\gamma}{a_{2}}, 0,0,0\right)
\end{aligned}
$$

\subsection{Existence and stability analysis of disease free equilibrium}

To find the Jacobian matrix of the model system, we differentiate equation (3.3) with respect to $x, y, T_{y}, R, B, I, T_{I}, R_{1}$ respectively to obtain.

$$
\begin{gathered}
\frac{d x^{*}}{d t}=\left[a_{1}-\beta \frac{a_{3} y}{\mu}\right] x^{*}+\left[-\beta x \frac{a_{3}}{\mu}\right] y^{*}+[b] R^{*} \\
\frac{d y^{*}}{d t}=\left[\beta \frac{a_{3} y}{\mu}\right] x^{*}+\left[\beta x \frac{a_{3}}{\mu}+\phi-\zeta-a_{1}-k \frac{a_{4} I}{\mu}-a_{6}-b_{4}\right] y^{*}+\left[-k \frac{a_{4} I}{\mu}\right] I^{*} \\
\frac{d T_{y}^{*}}{d t}=[\zeta] y^{*}+\left[-\left(a_{1}+d\right)\right] T_{y}^{*} \\
\frac{d R^{*}}{d t}=\left[b_{4}\right] y^{*}+\left[d_{1}\right] T_{y}^{*}+\left[-\left(a_{1}+b\right)\right] R^{*} \\
\frac{d B^{*}}{d t}=\left[-\alpha \frac{a_{4} I}{\mu}-a_{2}\right] B^{*}+\left[-\alpha \frac{a_{4} B}{\mu}\right] I^{*}+\left[b_{1}\right] R_{1}^{*} \\
\frac{d I^{*}}{d t}=\left[\alpha \frac{a_{4} I}{\mu}\right] B^{*}+\left[\alpha \frac{a_{4} B}{\mu}+\theta-d_{2}-a_{2}-a_{5}-b_{3}\right] I^{*} \\
\frac{d T_{I}^{*}}{d t}=\left[d_{2}\right] I^{*}+\left[-\left(d_{3}+a_{2}\right)\right] T_{I}^{*} \\
\frac{d R_{1}^{*}}{d t}=\left[d_{3}\right] T_{I}^{*}+\left[b_{3}\right] I^{*}+\left[-\left(a_{2}+b_{1}\right)\right] R_{1}^{*}
\end{gathered}
$$

We examine the stability of the disease free equilibrium using equation (3.16)

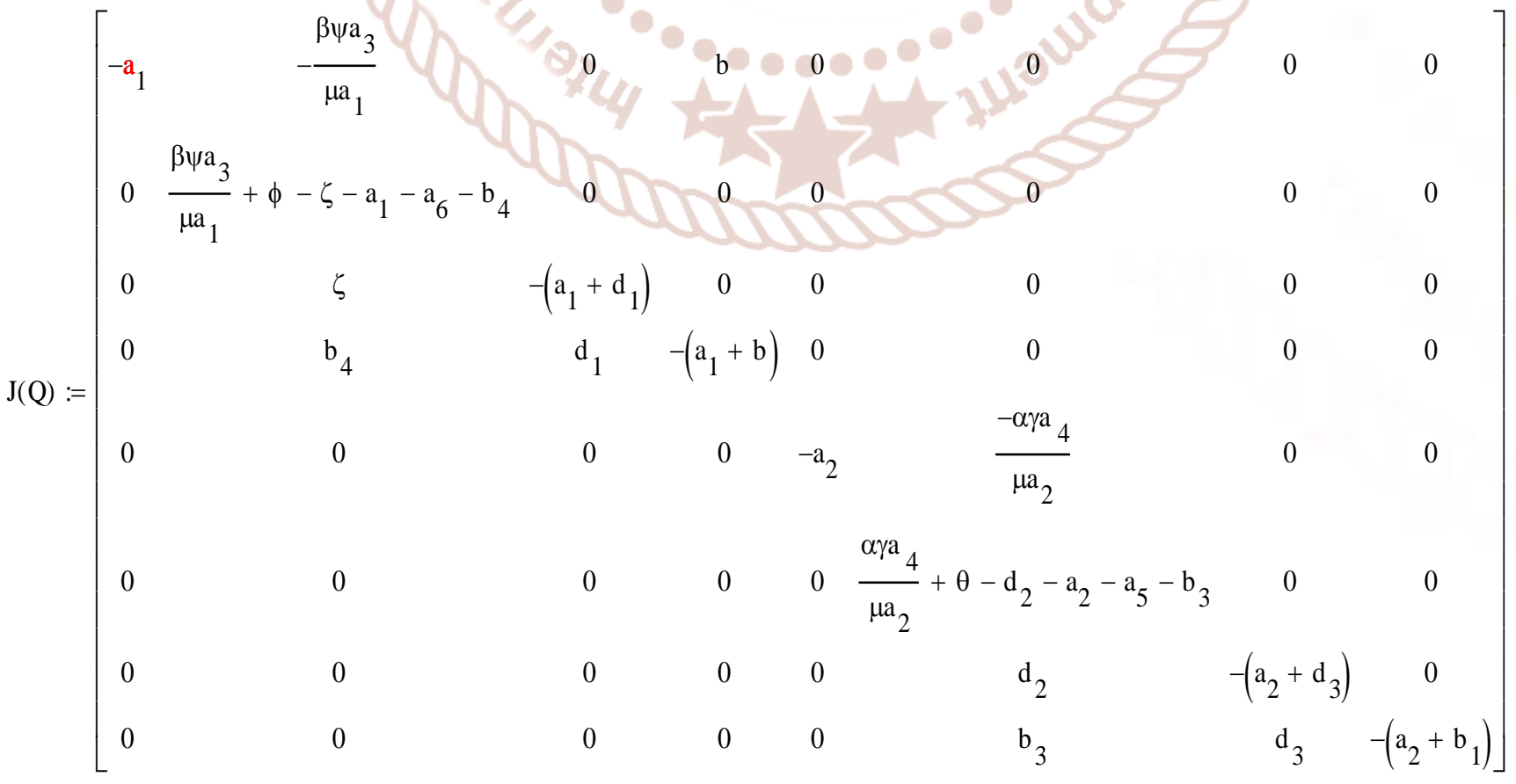


International Journal of Trend in Scientific Research and Development (IJTSRD) ISSN: 2456-6470

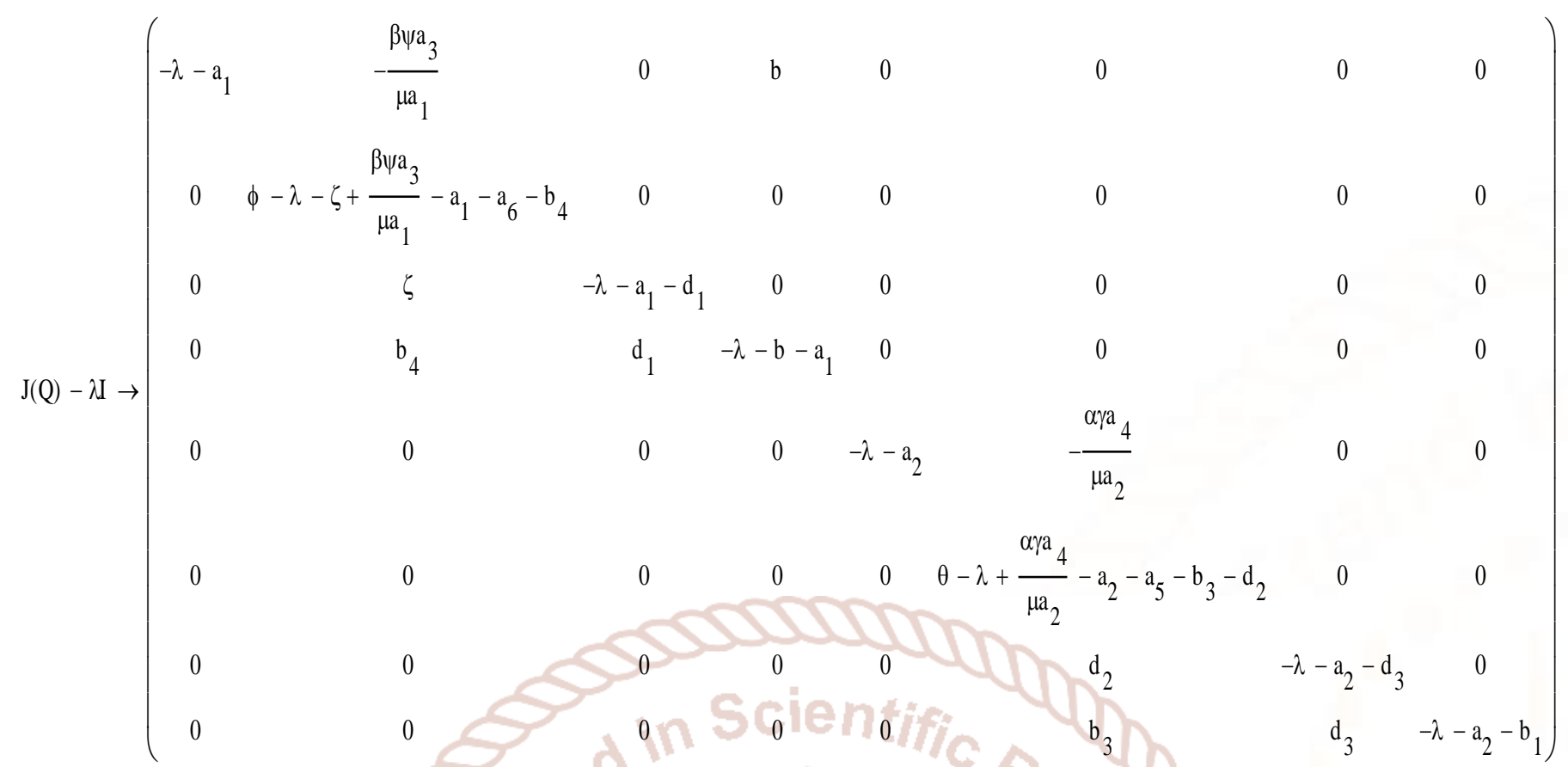

We need to show that all the eigen values of the matrix $J(Q)$ have negative real part. Observe that the first and fifth columns contain only the diagonal terms and this forms the two negative eigen values $\lambda_{1}=-a_{1}$ and $\lambda_{2}=$ $-a_{2}$, the other six eiginevalues can be obtained from the sub-matrix, $J_{2}(Q)$, formed by excluding the first and fifth rows and columns of $J(Q)$. thus, we have

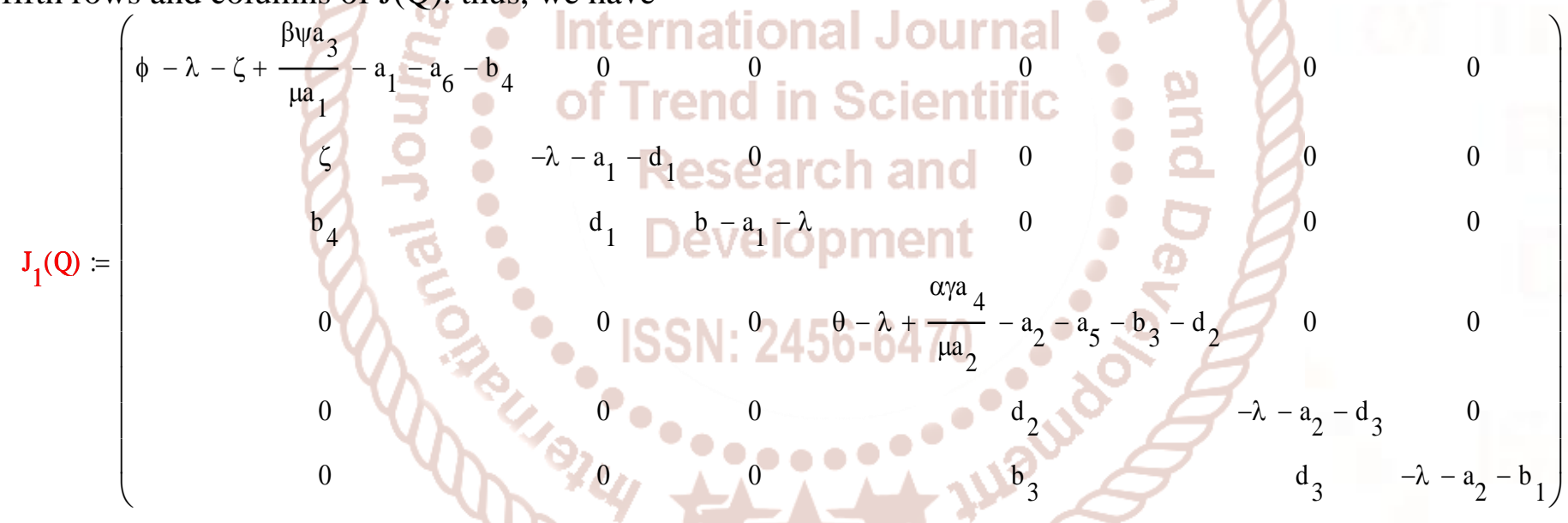

In the same way, the third and sixth column of $J_{1}(Q)$ contains only the diagonal term which forms negative eigenvalues $\lambda_{3}=-\left(a_{1}+b-b_{4}\right)$ and $\lambda_{4}=-\left(a_{2}+b_{1}\right)$. The remaining four eigenvalues are obtained from the sub - matrix

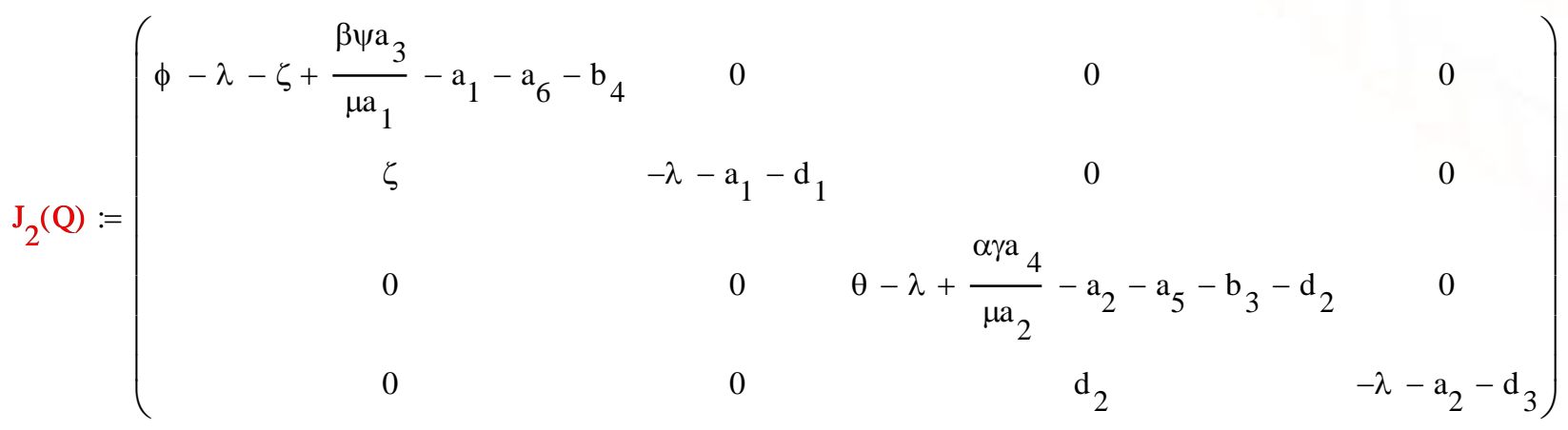


The eigenvalues of the matrix $J_{2}(Q)$ are the roots of the characteristic equation

$$
\begin{gathered}
\left(-\lambda+\frac{\beta \psi a_{3}}{\mu a_{1}}+\phi-\zeta-a_{1}-a_{6}-b_{4}\right)\left(-\lambda-a_{1}-d_{1}\right)\left(-\lambda+\frac{\alpha \gamma a_{4}}{\mu a_{2}}+\theta-d_{2}-a_{2}-a_{5}-b_{3}\right)\left(-\lambda-d_{3}\right. \\
\left.-a_{2}\right)=0
\end{gathered}
$$

which translates to

$$
\begin{gathered}
\lambda_{5}=-a_{1}-d_{1}, \lambda_{6}=-d_{3}-a_{2}, \lambda_{7}=\frac{\beta \psi a_{3}}{\mu a_{1}}+\phi-\zeta-a_{1}-a_{6}-b_{4}, \\
\text { and } \lambda_{8}=\frac{\alpha \gamma a_{4}}{\mu a_{2}}+\theta-d_{2}-a_{2}-a_{5}-b_{3}
\end{gathered}
$$

This implies that the eigenvalues $\lambda_{1,2, \ldots, 6}$ are both less that zero i.e., $\lambda_{1}<0, \lambda_{2}<0, \ldots, \lambda_{6}<0$.If $\frac{\beta \psi a_{3}}{\mu a_{1}}+\phi<$ $\zeta+a_{1}+a_{6}$ and $\frac{\alpha \gamma a_{4}}{\mu a_{2}}+\theta<d_{2}+a_{2}+a_{5}+b_{3}$, clearly, $\lambda_{7}$ and $\lambda_{8}$ will respectively be less than zero $\left(\lambda_{7}<0\right.$ and $\left.\lambda_{8}<0\right)$ and that means that the steady state is asymptotically stable. But if $\frac{\beta \psi a_{3}}{\mu a_{1}}+\phi>\zeta+a_{1}+$ $a_{6}$ and $\frac{\alpha \gamma a_{4}}{\mu a_{2}}+\theta>d_{2}+a_{2}+a_{5}+b_{3}, \lambda_{7}$ and $\lambda_{8}$ will respectively be greater than zero $\left(\lambda_{7}>0\right.$ and $\left.\lambda_{8}>0\right)$, we conclude that the steady state is unstable.

\subsection{Basic Reproduction Number $R_{0}$}

The basic reproduction number $R_{0}$ is the average number of secondary infectious infected by an infective individuals during the whole cause of disease in the case that all members of the population are susceptible (Zhien et al, 2009; Olaniyi and Obabiyi, 2013).

To obtain $R_{0}$ for model equation (3) we use the next generation technique (Van den Driessche and Watmough, 2002; Diekmann et al, 1990). We shall start with those equations of the model that describes the production of new infections and change in state among infected liver cells and red blood cells.

Let $H=\left[x, y, T_{y}, B, I, T_{I}\right]^{T}$ where $\mathrm{T}$ denotes transpose.

$$
\begin{gathered}
\frac{d H}{d t}=F(H)-V(H) \\
F(H)=\left[\begin{array}{c}
\frac{\beta \psi a_{3} y}{\mu a_{1}}+\phi y \\
0 \\
\frac{\alpha \gamma a_{4} I}{\mu a_{2}}+\theta I \\
0
\end{array}\right], V(H)=\left[\begin{array}{c}
\zeta y+a_{1} y+\frac{k y a_{4} I}{\mu}+a_{6} y+b_{4} y \\
-\zeta y+a_{1} T_{y}+d_{1} T_{y} \\
d_{2} I+a_{2} I+a_{5} I+b_{3} I \\
-d_{2} I+d_{3} T_{I}+a_{2} T_{I}
\end{array}\right]
\end{gathered}
$$

Finding the derivatives of $F$ and $V$ at the disease free equilibrium point $\Phi$ gives $F$ and $V$ respectively as

$$
\mathrm{F}:=\left(\begin{array}{cccc}
\frac{\beta \psi \mathrm{a}_{3}}{\mu \mathrm{a}_{1}}+\phi & 0 & 0 & 0 \\
0 & 0 & 0 & 0 \\
0 & 0 & \frac{\alpha \gamma \mathrm{a}_{4}}{\mu \mathrm{a}_{2}}+\theta & 0 \\
0 & 0 & 0 & 0
\end{array}\right) \quad \mathrm{V}:=\left(\begin{array}{ccccc}
\zeta+\mathrm{a}_{1}+\mathrm{a}_{6}+\mathrm{b}_{4} & 0 & 0 & 0 \\
-\zeta & \mathrm{a}_{1}+\mathrm{d}_{1} & 0 & 0 \\
0 & 0 & \mathrm{a}_{2}+\mathrm{a}_{5}+\mathrm{b}_{3}+\mathrm{d}_{2} & 0 \\
0 & 0 & -\mathrm{d}_{2} & \mathrm{a}_{2}+\mathrm{d}_{3}
\end{array}\right)
$$




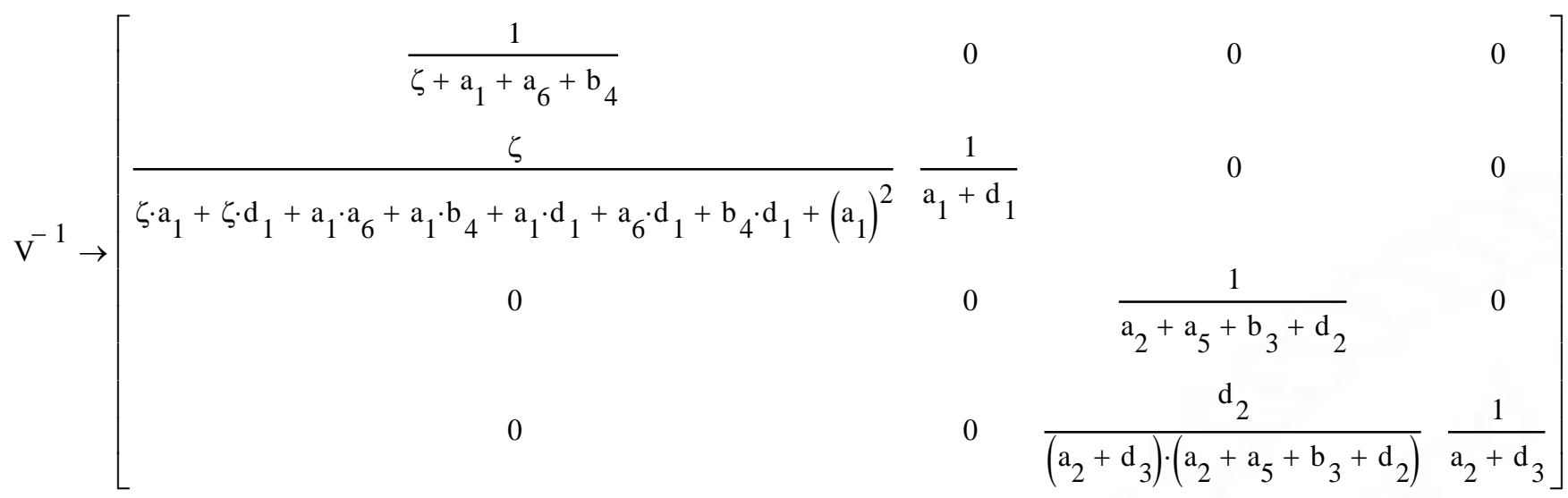

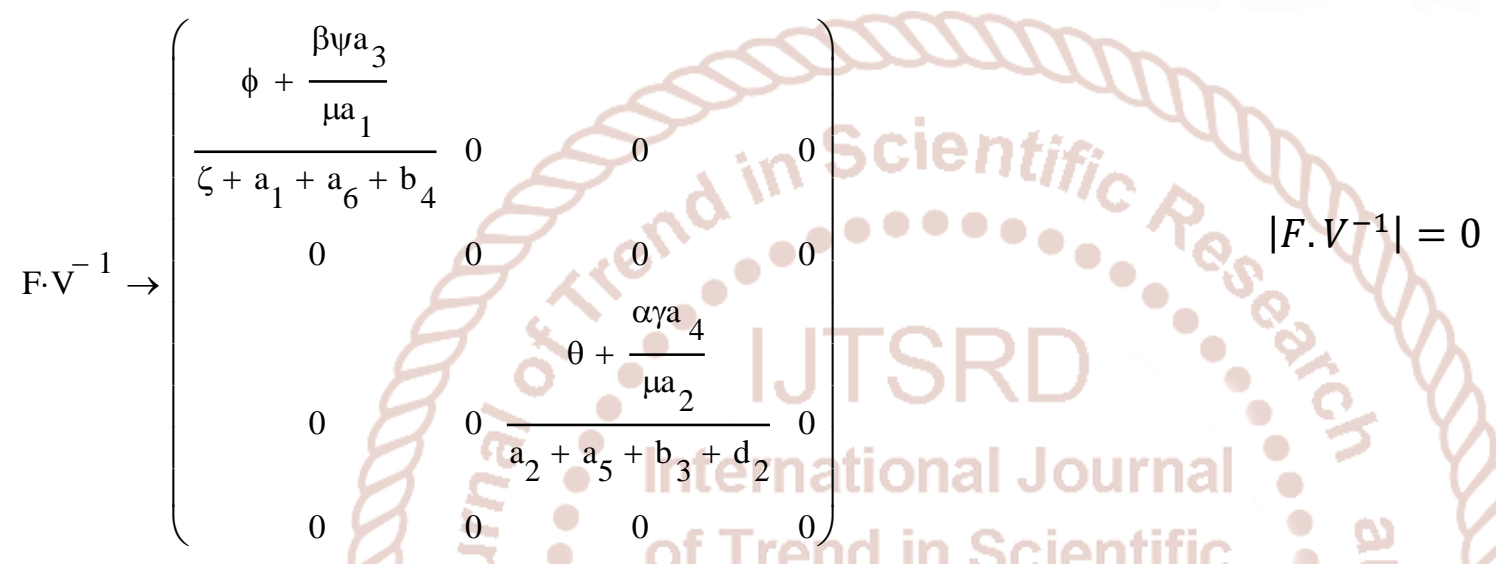

Here, we can obtain the basic reproduction number $R_{0}$ from the trce and determinant of the matrix $F V^{-1}=G$.

$$
R_{0}=W(G)=\frac{1}{2} \operatorname{trace}(G)+\sqrt{\operatorname{trace}(G)^{2}-4 \operatorname{det}(G)}
$$

Observe that $\operatorname{det}(G)=0$, so we have

$$
R_{0}=\frac{\beta \psi a_{3}}{a_{1} \mu\left(\zeta+a_{1}+a_{6}+b_{4}\right)}+\frac{\phi}{\zeta+a_{1}+a_{6}+b_{4}}+\frac{\gamma \alpha a_{4}}{a_{2} \mu\left(a_{2}+a_{5}+b_{3}+d_{2}\right)}+\frac{\theta}{a_{2}+a_{5}+b_{3}+d_{2}}
$$

From equation (3.19), $\frac{\beta \psi a_{3}}{a_{1} \mu}+\phi$ is the multiplication ability of the disease in the liver cells and the probability that an individual will move to the second level which is the infection of the red blood cells; $\frac{1}{\zeta+a_{1}+a_{6}+b_{4}}$ is the average duration of infectious period of the liver cells before the release of the parasite to invade red blood cells; $\frac{\gamma \alpha a_{4}}{a_{2} \mu}+\theta$ is the multiplication ability of the disease in the red blood cells and the probability that the individual will be infectious; $\frac{1}{a_{2}+a_{5}+b_{3}+d_{2}}$ is the average duration of the infectious period of the red blood cells. Let the basic reproduction number $R_{0}$ be written as

where

$$
R_{0}=R_{L}+R_{r}
$$

$$
\begin{aligned}
& R_{L}=\frac{\beta \psi a_{3}}{a_{1} \mu\left(\zeta+a_{1}+a_{6}+b_{4}\right)}+\frac{\phi}{\zeta+a_{1}+a_{6}+b_{4}} \\
& \text { and } \quad R_{r}=\frac{\gamma \alpha a_{4}}{a_{2} \mu\left(a_{2}+a_{5}+b_{3}+d_{2}\right)}+\frac{\theta}{a_{2}+a_{5}+b_{3}+d_{2}}
\end{aligned}
$$


We have $R_{L}$ describing the number of liver cells that one infectious liver cell infects over its expected infectious period in a completely susceptible liver cell population. While $R_{r}$ describes the number of red blood cells infected by one infectious red blood cell during the period of infectiousness in a completely susceptible red blood cell population.

\subsection{Existence of Endemic Equilibrium point}

Endemic equilibrium point describes the point at which the disease cannot totally be eradicated from the population. We shall show that the formulated model system (3.3) has an endemic point and we let $\Phi^{* *}$ be the endemic equilibrium point.

Theorem 2: the intracellular malaria model system (3.3) has no endemic equilibrium when $R_{0}<1$ but has a unique endemic equilibrium when $R_{0}>1$.

Proof: Let $\Phi^{* *}=\left(x^{* *}, y^{* *}, T_{y}^{* *}, R^{* *}, B^{* *}, I^{* *}, T_{I}^{* *}, R_{1}^{* *}\right)$ be a nontrivial equilibrium of the model system (3.3); i.e., all components of $\Phi^{* *}$ are positive. If we solve equation (3.3) simultaneously having in mind that $\Phi^{* *} \neq 0$ we have that

$$
\begin{aligned}
& d_{1} T_{y}^{* *}-a_{1} R^{* *}-b R^{* *}+b_{4} y^{* *}=0 \\
& a_{1}+b \\
& T_{y}^{* *}=\frac{\zeta y^{* *}}{a_{1}+d_{1}}
\end{aligned}
$$

Therefore (3.21) can be rewritten as

$$
\begin{aligned}
& R^{* *}=\frac{1}{a_{1}+b}\left(\frac{d_{1} \zeta y^{* *}}{a_{1}+d_{1}}+b_{4} y^{* *}\right) \\
& T_{I}^{* *}=\frac{d_{2} I^{* *}}{a_{2}+d_{3}} \\
& d_{2} I^{* *}-a_{2} T_{I}^{* *}-d_{3} T_{I}^{* *}=0 \\
& \frac{d_{2} d_{3} I^{* *}}{a_{2}+d_{3}}+b_{3} I^{* *}=\left(a_{2}+b_{1}\right) R_{1}^{* *} \\
& R_{1}^{* *}=\frac{\left[d_{2} d_{3}+b_{3}\left(a_{2}+d_{3}\right)\right] I^{* *}}{\left(a_{2}+b_{1}\right)\left(a_{2}+d_{3}\right)} \\
& \alpha B^{* *} \frac{a_{4} I^{* *}}{\mu}+\theta I^{* *}-d_{2} I^{* *}-a_{2} I^{* *}-a_{5} I^{* *}-b_{3} I^{* *}=0 \\
& \alpha B^{* *} \frac{a_{4}}{\mu}+\theta-d_{2}-a_{2}-a_{5}-b_{3}=0 \\
& B^{* *}=\frac{\left(d_{2}+a_{2}+a_{5}+b_{3}-\theta\right) \mu}{\alpha a_{4}} \\
& \gamma-\alpha\left(\frac{\left(d_{2}+a_{2}+a_{5}+b_{3}-\theta\right) \mu}{\alpha a_{4}}\right) \frac{a_{4} I^{* *}}{\mu}-a_{2}\left(\frac{\left(d_{2}+a_{2}+a_{5}+b_{3}-\theta\right) \mu}{\alpha a_{4}}\right) \\
& +b_{1} \frac{\left[d_{2} d_{3}+b_{3}\left(a_{2}+d_{3}\right)\right] I^{* *}}{\left(a_{2}+b_{1}\right)\left(a_{2}+d_{3}\right)}=0 \\
& b_{1}\left[d_{2} d_{3}+b_{3}\left(a_{2}+d_{3}\right)\right] I^{* *}-\left(d_{2}+a_{2}+a_{5}+b_{3}-\theta\right)\left(a_{2}+b_{1}\right)\left(a_{2}+d_{3}\right) I^{* *} \\
& =\left[a_{2}\left(\frac{\left(d_{2}+a_{2}+a_{5}+b_{3}-\theta\right) \mu}{\alpha a_{4}}\right)-\gamma\right]\left(a_{2}+b_{1}\right)\left(a_{2}+d_{3}\right)
\end{aligned}
$$




$$
\begin{aligned}
& I^{* *}=\frac{\left[a_{2}\left(\frac{\left(d_{2}+a_{2}+a_{5}+b_{3}-\theta\right) \mu}{\alpha a_{4}}\right)-\gamma\right]\left(a_{2}+b_{1}\right)\left(a_{2}+d_{3}\right)}{b_{1}\left[d_{2} d_{3}+b_{3}\left(a_{2}+d_{3}\right)\right]-\left(d_{2}+a_{2}+a_{5}+b_{3}-\theta\right)\left(a_{2}+b_{1}\right)\left(a_{2}+d_{3}\right)} \\
& \beta x \frac{a_{3} y^{* *}}{\mu}+\phi y^{* *}-\zeta y^{* *}-a_{1} y^{* *}-a_{6} y^{* *}-k_{y} \frac{a_{4} I^{* *}}{\mu}-b_{4} y^{* *}=0 \\
& \beta x^{* *} \frac{a_{3}}{\mu}+\phi-\zeta-a_{1}-a_{6}-k_{y} \frac{a_{4} I^{* *}}{\mu}-b_{4}=0 \\
& x^{* *}=\left(\zeta+a_{1}+a_{6}+k_{y} \frac{a_{4} I^{* *}}{\mu}+b_{4}-\phi\right) \frac{\mu}{\beta a_{3}} \\
& \psi-a_{1} x^{* *}-\beta x^{* *} \frac{a_{3} y^{* *}}{\mu}+b R^{* *}=0 \\
& \psi-a_{1}\left(\zeta+a_{1}+a_{6}+k_{y} \frac{a_{4} I^{* *}}{\mu}+b_{4}-\phi\right) \frac{\mu}{\beta a_{3}}-\beta\left(\zeta+a_{1}+a_{6}+k_{y} \frac{a_{4} I^{* *}}{\mu}+b_{4}-\phi\right) \frac{\mu}{\beta a_{3}} \frac{a_{3} y^{* *}}{\mu} \\
& +\frac{b}{a_{1}+b}\left(\frac{d_{1} \zeta y^{* *}}{a_{1}+d_{1}}+b_{4}\right)=0 \\
& \beta\left(\zeta+a_{1}+a_{6}+k_{y} \frac{a_{4} I^{* *}}{\mu}+b_{4}-\phi\right) \frac{\mu}{\beta a_{3}} \frac{a_{3} y^{* *}}{\mu}-\frac{b}{a_{1}+b}\left(\frac{d_{1} \zeta}{a_{1}+d_{1}}+b_{4}\right) y^{* *} \\
& =\left[\psi-a_{1}\left(\zeta+a_{1}+a_{6}+k_{y} \frac{a_{4} I^{* *}}{\mu}+b_{4}-\phi\right) \frac{\mu}{\beta a_{3}}\right] \\
& y^{* *}=\frac{\left[\psi-a_{1}\left(\zeta+a_{1}+a_{6}+k_{y} \frac{a_{4} I^{* *}}{\mu}+b_{4}-\phi\right) \frac{\mu}{\beta a_{3}}\right]}{\left(\zeta+a_{1}+a_{6}+k_{y} \frac{a_{4} I^{* *}}{\mu}+b_{4}-\phi\right)-\frac{b}{a_{1}+b}\left(\frac{d_{1} \zeta}{a_{1}+d_{1}}+b_{4}\right)}
\end{aligned}
$$

\subsection{Sensitivity Analysis of the Basic Reproduction Number $\boldsymbol{R}_{0}$}

Observe that the basic reproduction number $R_{0}$ is in the form $R_{0}=R_{L}+R_{r}$, where $R_{L}$ and $R_{r}$ are functions of nine parameters respectively. But $R_{0}$ is a function of sixteen parameters which comprises of the basic reproduction number at the liver site and the basic reproduction number at the blood site. To control the disease, these parameter values must control $R_{0}$, such that its value will be less than one $\left(R_{0}<1\right)$. Therefore change in the parameter values, results in change in $R_{0}$ and if we let

$$
q_{L}=\left(\beta, \psi, a_{1}, a_{3}, a_{6}, \mu, \zeta, \phi\right) \text { and } q_{r}=\left(\gamma, \alpha, a_{2}, a_{4}, a_{5}, \mu, b_{3}, d_{2}, \theta\right)
$$

then the rate of change of $R_{0}$ for a change in the value of parameter $q$ can be estimated from a normalized sensitivity index

$$
\begin{gathered}
\mathrm{Z}_{q}^{R_{0}}=\frac{\partial R_{L}}{\partial q_{L}} \cdot \frac{R_{L}}{q_{L}}+\frac{\partial R_{r}}{\partial q_{r}} \cdot \frac{R_{r}}{q_{r}} \\
\mathrm{Z}_{q_{L}}^{R_{L}}=\frac{\partial R_{L}}{\partial q_{L}} \cdot \frac{R_{L}}{q_{L}} \text { and } \mathrm{Z}_{q_{r}}^{R_{r}}=\frac{\partial R_{r}}{\partial q_{r}} \cdot \frac{R_{r}}{q_{r}}
\end{gathered}
$$


International Journal of Trend in Scientific Research and Development (IJTSRD) ISSN: 2456-6470

Table 1 Parameter values for calculating $R_{0}$ and Numerical Simulation of the Model

\begin{tabular}{|c|c|c|c|}
\hline Parameters & Description & $\begin{array}{l}\text { Value } \\
\text { Range }\end{array}$ & Reference \\
\hline$\psi$ & Recruitment levelof uninfected hepatocytes & $3 \times 10^{8}$ & Mota et al, 2001 \\
\hline$a_{1}$ & $\begin{array}{l}\text { Natural death rate of both uninfected, infected, } \\
\text { treated and recovered hepatocytes }\end{array}$ & $\begin{array}{l}0.002- \\
0.0067\end{array}$ & Mota et al, 2001 \\
\hline$\beta$ & Rate at which hepatocytes are infected & $4 \times 10^{-9}$ & Tabo et al, 2017 \\
\hline$\mu$ & Natural death rate of malaria parasite & 48 & Tabo et al, 2017 \\
\hline$a_{3}$ & $\begin{array}{l}\text { Rate at which infected hepatocytes produce free } \\
\text { sporozoites }\end{array}$ & 0.181 & Esteva et al, 2009 \\
\hline$b$ & $\begin{array}{l}\text { Rate at which recovered hepatocytes move to } \\
\text { susceptible class }\end{array}$ & $\begin{array}{l}1.3 \\
\times 10^{-4} \\
\end{array}$ & $\begin{array}{l}\text { Chitnis, 2008; Mohammed and } \\
\text { Orukpe, } 2014\end{array}$ \\
\hline$\zeta$ & Treatment rate of infectious hepatocytes & 0.95 & $\begin{array}{l}\text { Mohammed and Orukpe, 2014; } \\
\text { Castillo-Riquelme et al, } 2008 .\end{array}$ \\
\hline$d_{1}$ & $\begin{array}{c}\text { Movement rate of treated infectious hepatocytes } \\
\text { to recovered class }\end{array}$ & & Ducrot et al, 2008 \\
\hline$a_{2}$ & $\begin{array}{c}\begin{array}{c}\text { Natural death rate of erythrocytes (Red blood } \\
\text { cells) }\end{array} \\
\end{array}$ & & Anderson et al, 1989 \\
\hline$\gamma$ & Recruitment level of erythrocytes & $2.5 \times 10^{8}$ & Austin et al, 1998 \\
\hline$\alpha$ & $\begin{array}{l}\text { Rate at which uninfected erythrocytes are being } \\
\text { infected }\end{array}$ & $2 \times 10^{1}$ & Dondorp et al, 2000 \\
\hline$a_{4}$ & $\begin{array}{l}\text { Rate at which infected erythrocytes produce free } \\
\text { merozoites }\end{array}$ & 16 & Chiyaka et al, 2010 \\
\hline$a_{5}$ & $\begin{array}{c}\text { Disease induced death rate of infected } \\
\text { erythrocytes }\end{array}$ & 0.24 & Chiyaka et al, 2010 \\
\hline$a_{6}$ & $\begin{array}{l}\text { Disease induced death rate of infected } \\
\text { hepatocytes }\end{array}$ & 2.0 & Tabo et al, 2017 \\
\hline$\phi$ & $\begin{array}{c}\text { The rate at which infected hepatocytes } \\
\text { proliferate }\end{array}$ & $3 \times 10^{-}$ & Estimated \\
\hline$\theta$ & $\begin{array}{l}\text { The rate at which infected erythrocytes } \\
\text { proliferate }\end{array}$ & $\begin{array}{l}2.5 \\
\times 10^{-5} \\
\end{array}$ & Estimated \\
\hline$d_{2}$ & $\begin{array}{c}\text { Rate at which infectederythrocytesare being } \\
\text { treated }\end{array}$ & & Mohammed and Orukpe, 2014 \\
\hline$d_{3}$ & $\begin{array}{c}\text { Movement rate of treated infected erythrocytes } \\
\text { to recovered class }\end{array}$ & & Ducrot et al, 2008 \\
\hline$k_{y}$ & $\begin{array}{l}\text { Rate at which infected hepatocytes produce } \\
\text { merozoites (malaria parasite) }\end{array}$ & & $\begin{array}{l}\text { Tabo et al, 2017; Chiyaka et al, } \\
2010\end{array}$ \\
\hline$b_{3}$ & Recovered erythrocytes due to immune response & 4.56 & Estimated \\
\hline$b_{4}$ & Recovered hepatocytes due to immune response & 0.0035 & Shah and Gupta, 2013 \\
\hline$b_{1}$ & $\begin{array}{l}\text { Movement rate of recovered erythrocytes to } \\
\text { susceptible class class }\end{array}$ & $\begin{array}{l}1.37 \\
\times 10^{-4} \\
\end{array}$ & $\begin{array}{l}\text { Chitnis, 2008; Molineaux and } \\
\text { Gramiccia, } 1980 \\
\end{array}$ \\
\hline
\end{tabular}

To calculate the value of $R_{0}$, we use the parameters as stated in table 1 .

$$
\begin{gathered}
R_{0}=R_{L}+R_{r} \\
R_{L}=\frac{\beta \psi a_{3}}{a_{1} \mu\left(\zeta+a_{1}+a_{6}+b_{4}\right)}+\frac{\phi}{\zeta+a_{1}+a_{6}+b_{4}} \\
R_{r}=\frac{\gamma \alpha a_{4}}{a_{2} \mu\left(a_{2}+a_{5}+b_{3}+d_{2}\right)}+\frac{\theta}{a_{2}+a_{5}+b_{3}+d_{2}}
\end{gathered}
$$


International Journal of Trend in Scientific Research and Development (IJTSRD) ISSN: 2456-6470

$$
\begin{gathered}
R_{L}=\frac{4 \times 10^{-9} \times 3 \times 10^{8} \times 0.181}{0.004 \times 48(0.95+0.004+2+0.0035)}+\frac{3 \times 10^{-5}}{0.95+0.004+2+0.0035} \\
=0.382512257 \\
R_{r}=\frac{2.5 \times 10^{8} \times 2 \times 10^{-10} \times 16}{0.0083 \times 48(0.0083+0.24+4.56+0.95)}+\frac{2.5 \times 10^{-5}}{0.0083+0.24+4.56+0.95} \\
R_{0}=0.348723952
\end{gathered}
$$

The normalized sensitivity index of the basic reproduction number with respect to $\beta, \psi, a_{1}, a_{3}$, $a_{6}, \mu, \zeta, \phi$ is given by

$$
\begin{aligned}
& \mathrm{Z}_{q_{L}}^{R_{L}}=\frac{\partial R_{L}}{\partial q_{L}} \cdot \frac{R_{L}}{q_{L}} \\
& \mathrm{Z}_{\beta}^{R_{L}}=\frac{\partial R_{L}}{\partial \beta} \cdot \frac{R_{L}}{\beta}=\left(\frac{\psi a_{3}}{a_{1} \mu\left(\zeta+a_{1}+a_{6}+b_{4}\right)}\right)\left(\frac{R_{L}}{\beta}\right)=9.0110964 \times 10^{15} \\
& \mathrm{Z}_{\psi}^{R_{L}}=\frac{\partial R_{L}}{\partial \psi} \cdot \frac{R_{L}}{\psi}=\left(\frac{\beta a_{3}}{a_{1} \mu\left(\zeta+a_{1}+a_{6}+b_{4}\right)}\right)\left(\frac{R_{L}}{\psi}\right)=1.69 \times 10^{-18} \\
& \mathrm{Z}_{a_{3}}^{R_{L}}=\frac{\partial R_{L}}{\partial a_{3}} \cdot \frac{R_{L}}{a_{3}}=\left(\frac{\beta \psi}{a_{1} \mu\left(\zeta+a_{1}+a_{6}+b_{4}\right)}\right)\left(\frac{R_{L}}{a_{3}}\right)=4.4018101352 \\
& \mathrm{Z}_{\phi}^{R_{L}}=\frac{\partial R_{L}}{\partial \phi} \cdot \frac{R_{L}}{\phi}=\left(\frac{1}{\zeta+a_{1}+a_{6}+b_{4}}\right)\left(\frac{R_{L}}{\phi}\right)=4311.2116885 \\
& \mathrm{Z}_{a_{1}}^{R_{L}}=\frac{\partial R_{L}}{\partial a_{1}} \cdot \frac{R_{L}}{a_{1}}=-\left(\frac{\beta \psi a_{3}\left(\zeta+2 a_{1}+a_{6}+b_{4}\right)}{\left(a_{1} \mu \zeta+a_{1}^{2} \mu+a_{1} \mu a_{6}+a_{1} \mu b_{4}\right)^{2}}+\frac{\phi}{\left(\zeta+a_{1}+a_{6}+b_{4}\right)^{2}}\right)\left(\frac{R_{L}}{a_{1}}\right) \\
& =-9156.8606658 \\
& \mathrm{Z}_{\mu}^{R_{L}}=\frac{\partial R_{L}}{\partial \mu} \cdot \frac{R_{L}}{\mu}=-\left(\frac{\beta \psi a_{3}}{a_{1} \mu^{2}\left(\zeta+a_{1}+a_{6}+b_{4}\right)}\right)\left(\frac{R_{L}}{\mu}\right)=-0.0488762883 \\
& \mathrm{Z}_{\zeta}^{R_{L}}=\frac{\partial R_{L}}{\partial \zeta} \cdot \frac{R_{L}}{\zeta}=-\left(\frac{\beta \psi a_{3}}{a_{1} \mu\left(\zeta+a_{1}+a_{6}+b_{4}\right)^{2}}+\frac{\phi}{\left(\zeta+a_{1}+a_{6}+b_{4}\right)^{2}}\right)\left(\frac{R_{L}}{\zeta}\right) \\
& =-0.0521115791 \\
& \mathrm{Z}_{b_{4}}^{R_{L}}=\frac{\partial R_{L}}{\partial b_{4}} \cdot \frac{R_{L}}{b_{4}}=-\left(\frac{\beta \psi a_{3}}{a_{1} \mu\left(\zeta+a_{1}+a_{6}+b_{4}\right)^{2}}+\frac{\phi}{\left(\zeta+a_{1}+a_{6}+b_{4}\right)^{2}}\right)\left(\frac{R_{L}}{b_{4}}\right) \\
& =-14.144571463 \\
& \mathrm{Z}_{a_{6}}^{R_{L}}=\frac{\partial R_{L}}{\partial a_{6}} \cdot \frac{R_{L}}{a_{6}}=-\left(\frac{\beta \psi a_{3}}{a_{1} \mu\left(\zeta+a_{1}+a_{6}+b_{4}\right)^{2}}+\frac{\phi}{\left(\zeta+a_{1}+a_{6}+b_{4}\right)^{2}}\right)\left(\frac{R_{L}}{a_{6}}\right) \\
& =-0.0247530001
\end{aligned}
$$


International Journal of Trend in Scientific Research and Development (IJTSRD) ISSN: 2456-6470

The normalized sensitivity index of the basic reproduction number with respect to $\gamma, \alpha, a_{2}, a_{4}, a_{5}, \mu, b_{3}, d_{2}, \theta$ is given by

$$
\begin{aligned}
& \mathrm{Z}_{q_{L}}^{R}=\frac{\partial R_{r}}{\partial q_{r}} \cdot \frac{R_{r}}{q_{r}} \\
& \mathrm{Z}_{\gamma}^{R_{r}}=\frac{\partial R_{r}}{\partial \gamma} \cdot \frac{R_{r}}{\gamma}=\left(\frac{\alpha a_{4}}{a_{2} \mu\left(a_{2}+a_{5}+b_{3}+d_{2}\right)}\right)\left(\frac{R_{r}}{\gamma}\right)=1.96 \times 10^{-18} \\
& \mathrm{Z}_{\alpha}^{R_{r}}=\frac{\partial R_{r}}{\partial \alpha} \cdot \frac{R_{r}}{\alpha}=\left(\frac{\gamma a_{4}}{a_{2} \mu\left(a_{2}+a_{5}+b_{3}+d_{2}\right)}\right)\left(\frac{R_{r}}{\alpha}\right)=3.0401721 \times 10^{18} \\
& \mathrm{Z}_{a_{4}}^{R_{r}}=\frac{\partial R_{r}}{\partial a_{4}} \cdot \frac{R_{r}}{a_{4}}=\left(\frac{\gamma \alpha}{a_{2} \mu\left(a_{2}+a_{5}+b_{3}+d_{2}\right)}\right)\left(\frac{R_{r}}{a_{4}}\right)=0.0004750269 \\
& \mathrm{Z}_{\theta}^{R_{r}}=\frac{\partial R_{r}}{\partial \theta} \cdot \frac{R_{r}}{\theta}=\left(\frac{1}{\left(a_{2}+a_{5}+b_{3}+d_{2}\right)}\right)\left(\frac{R_{r}}{\theta}\right)=2422.4090582 \\
& \mathrm{Z}_{a_{2}}^{R_{L}}=\frac{\partial R_{r}}{\partial a_{2}} \cdot \frac{R_{r}}{a_{2}}=-\left(\frac{\gamma \alpha a_{4}\left(2 a_{2}+a_{5}+b_{3}+d_{2}\right)}{\left(a_{2}^{2} \mu+a_{2} a_{5} \mu+a_{2} b_{3} \mu+a_{2} d_{2} \mu\right)^{2}}+\frac{\theta}{\left(a_{2}+a_{5}+b_{3}+d_{2}\right)^{2}}\right)\left(\frac{R_{r}}{a_{2}}\right) \\
& =-1761.6460452 \\
& \mathrm{Z}_{\mu}^{R_{r}}=\frac{\partial R_{r}}{\partial \mu} \cdot \frac{R_{r}}{\mu}=-\left(\frac{\gamma \alpha a_{4}}{a_{2} \mu^{2}\left(a_{2}+a_{5}+b_{3}+d_{2}\right)}\right)\left(\frac{R_{r}}{\mu}\right)=-0.0000527808 \\
& \mathrm{Z}_{a_{5}}^{R_{r}}=\frac{\partial R_{r}}{\partial a_{5}} \cdot \frac{R_{r}}{a_{5}}=-\left(\frac{\gamma \alpha a_{4}}{a_{2} \mu\left(a_{2}+a_{5}+b_{3}+d_{2}\right)^{2}}+\frac{\theta}{\left(a_{2}+a_{5}+b_{3}+d_{2}\right)^{2}}\right)\left(\frac{R_{r}}{a_{5}}\right) \\
& =-0.0879950061 \\
& \mathrm{Z}_{b_{3}}^{R_{r}}=\frac{\partial R_{r}}{\partial b_{3}} \cdot \frac{R_{r}}{b_{3}}=-\left(\frac{\gamma \alpha a_{4}}{a_{2} \mu\left(a_{2}+a_{5}+b_{3}+d_{2}\right)^{2}}+\frac{\theta}{\left(a_{2}+a_{5}+b_{3}+d_{2}\right)^{2}}\right)\left(\frac{R_{r}}{b_{3}}\right) \\
& =-0.0046313161 \\
& \mathrm{Z}_{a_{5}}^{R_{r}}=\frac{\partial R_{r}}{\partial d_{2}} \cdot \frac{R_{r}}{d_{2}}=-\left(\frac{\gamma \alpha a_{4}}{a_{2} \mu\left(a_{2}+a_{5}+b_{3}+d_{2}\right)^{2}}+\frac{\theta}{\left(a_{2}+a_{5}+b_{3}+d_{2}\right)^{2}}\right)\left(\frac{R_{r}}{d_{2}}\right) \\
& =-0.00222303174
\end{aligned}
$$

Table 2: The effect of the parameters on $R_{L}$.

\begin{tabular}{|c|c|c|}
\hline Parameters & Value Range & Effect on $\boldsymbol{R}_{01}$ \\
\hline$\beta$ & $4 \times 10^{-9}$ & $9.0110964 \times 10^{15}$ \\
\hline$\psi$ & $3 \times 10^{8}$ & $1.69 \times 10^{-18}$ \\
\hline$a_{3}$ & 0.181 & 4.4018101352 \\
\hline$\phi$ & $3 \times 10^{-5}$ & 4311.2116885 \\
\hline$a_{1}$ & 0.004 & -9156.8606658 \\
\hline$\mu$ & 48 & -0.0488762883 \\
\hline$\zeta$ & 0.95 & -0.0521115791 \\
\hline$b_{4}$ & 0.0035 & -14.144571463 \\
\hline$a_{6}$ & 2.0 & -0.0247530001 \\
\hline
\end{tabular}


Table 3: The effect of the parameters on $R_{r}$.

\begin{tabular}{|c|c|c|}
\hline Parameters & Value Range & Effect on $\boldsymbol{R}_{01}$ \\
\hline$\gamma$ & $2.5 \times 10^{8}$ & $1.69 \times 10^{-18}$ \\
\hline$\alpha$ & $2 \times 10^{-10}$ & $3.0401721 \times 10^{18}$ \\
\hline$a_{4}$ & 16 & 0.0004750269 \\
\hline$\theta$ & $2.5 \times 10^{-5}$ & 2422.4090582 \\
\hline$a_{2}$ & 0.0083 & -1761.6460452 \\
\hline$\mu$ & 48 & -0.0000527808 \\
\hline$a_{5}$ & 0.24 & -0.0879950061 \\
\hline$b_{3}$ & 4.56 & -0.0046313161 \\
\hline$d_{2}$ & 0.95 & -0.0222303174 \\
\hline
\end{tabular}

The sensitivity index $Z(\beta), Z(\psi), Z\left(a_{3}\right)$ and $Z(\phi)$ are all positive and this shows that the value of $R_{L}$ increases as the value of $\beta, \psi, a_{3}$ and $\phi$ increases. The remaining indices $Z\left(a_{1}\right), Z\left(b_{4}\right), Z\left(a_{6}\right), Z(\mu)$ and $Z(\zeta)$ are negative, indicating that the value $R_{L}$ decreases as $a_{1}, b_{4}, a_{6}, \mu$ and $\zeta$ increases. Actually, the effectiveness of control may be measured by its effect on $R_{L}$. if the reduction in $R_{L}<1$ can be maintained by the parameters $a_{1}, b_{4}, a_{6}, \mu$ and $\zeta$, then it will reduce the endemicity of the disease. This implies that these parameters can help in reducing the rate of malaria infection over time in the liver and if it is maintained, the transmission of the disease may decrease, causing the cases in the liver population to go below an endemicity threshold.

Similarly, the sensitivity index $Z(\gamma), Z(\alpha), Z\left(a_{4}\right)$ and $Z(\theta)$ are all positive indicating that the value of $R_{r}$ increases as the value of $\gamma, \alpha, a_{4}$ and $\theta$ increases. The indices of remaining parameters $Z\left(a_{2}\right), Z\left(b_{3}\right), Z\left(a_{5}\right), Z(\mu)$ and $Z\left(d_{2}\right)$ are negative, and this shows that the value of $R_{r}$ decreases as $a_{2}, b_{3}, a_{5}, \mu$ and $d_{2}$ increases. Since the effectiveness of control may be measured by its effect on $R_{r}$ and if the reduction in $R_{r}<1$ can be maintained by the parameters $a_{1}, b_{4}, a_{6}, \mu$ and $\zeta$, then endemicity of the disease in the erythrocyte will be reduced. Therefore, the parameters $a_{1}, b_{4}, a_{6}, \mu$ and $\zeta$ can help in reducing the rate of malaria infection over time in the erythrocyte and if maintained, the transmission of the disease may decrease, causing the cases in the erythrocyte population to drop beyond the endemicity threshold.

\section{Numerical Analysis and Results}

The numerical behavior of system (3.3) were studied using the parameter values given in table 1 and by considering initial conditions, $\varphi=\left\{x(0), y(0), T_{y}(0), R(0), B(0), I(0), T_{I}(0), R_{1}(0)\right\}$. The multiplication ability of meroziote in the hepatocyte is $\frac{\beta \psi a_{3}}{a_{1} \mu}+\phi=1.13128$, while the probability that the red blood cell will be infected by sporozoites is $\frac{1}{\zeta+a_{1}+a_{6}+b_{4}}=0.3381234151$. Also, The multiplication ability of sporoziote in the erythrocyte is $\frac{\gamma \alpha a_{4}}{a_{2} \mu}+\theta=2.0080571285$, while the probability that the human host will be infectious is $\frac{1}{a_{2}+a_{5}+b_{3}+d_{2}}=0.1736623656$.

The numerical simulation are conducted using Matlab software and the results are given in figure $2-4$ where figures $2 \mathrm{i}-2 \mathrm{iii}$ illustrate the behavior of the reproductive number $R_{L}$ for different values of the model parameter $b_{4}$ and $b_{3}$ respectively. Figures $3 \mathrm{i}-3$ iii also show the behavior of the reproductive number $R_{r}$ for different values of the model parameter $\zeta$ and $d_{2}$ respectively where $\zeta$ is represented with $g_{4}$. Lastly, figures $4 \mathrm{i}-4 \mathrm{viii}$ and $5 \mathrm{i}-5$ viii show the varying effects of the immune system and treatment controls.

The basic reproduction number of the system is given by

$$
R_{0}=R_{L}+R_{r}=0.731236209<1
$$


indicating that the basic reproduction number is less than one. Therefore, the disease free equilibrium is stable showing that malaria infection can be controlled in the population using adequate treatment method. However, it also confirms the result of the sensitivity analysis of $R_{L}$ and $R_{r}$ in tables 2 and 3 respectively. We then state that with effective treatment of infectious human, the future number of malaria infection cases will reduce in the population.

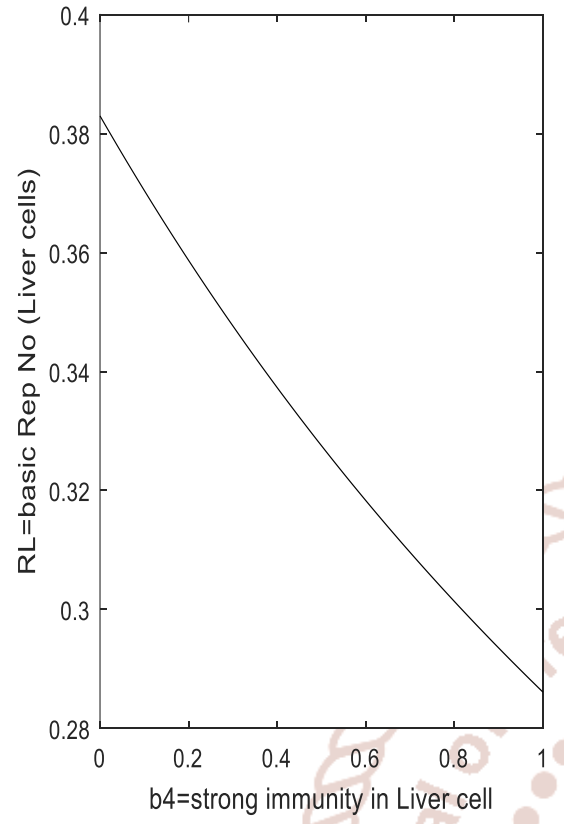

fig $2 \mathrm{i}$

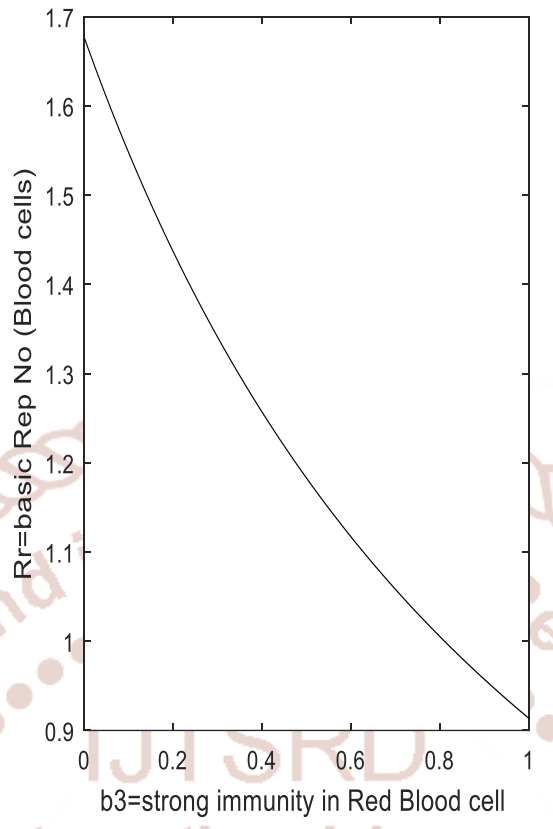

fig $2 \mathrm{ii}$

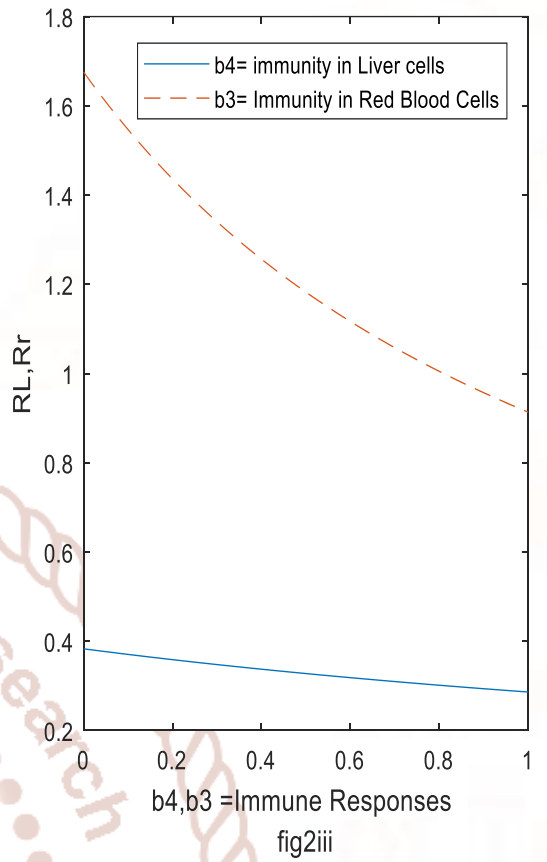

Figures (2i - 2iii): Numerical Simulation of the Basic Reproduction Number R_L and R_r using different rate of b_4 and b_3, (Immune Control)
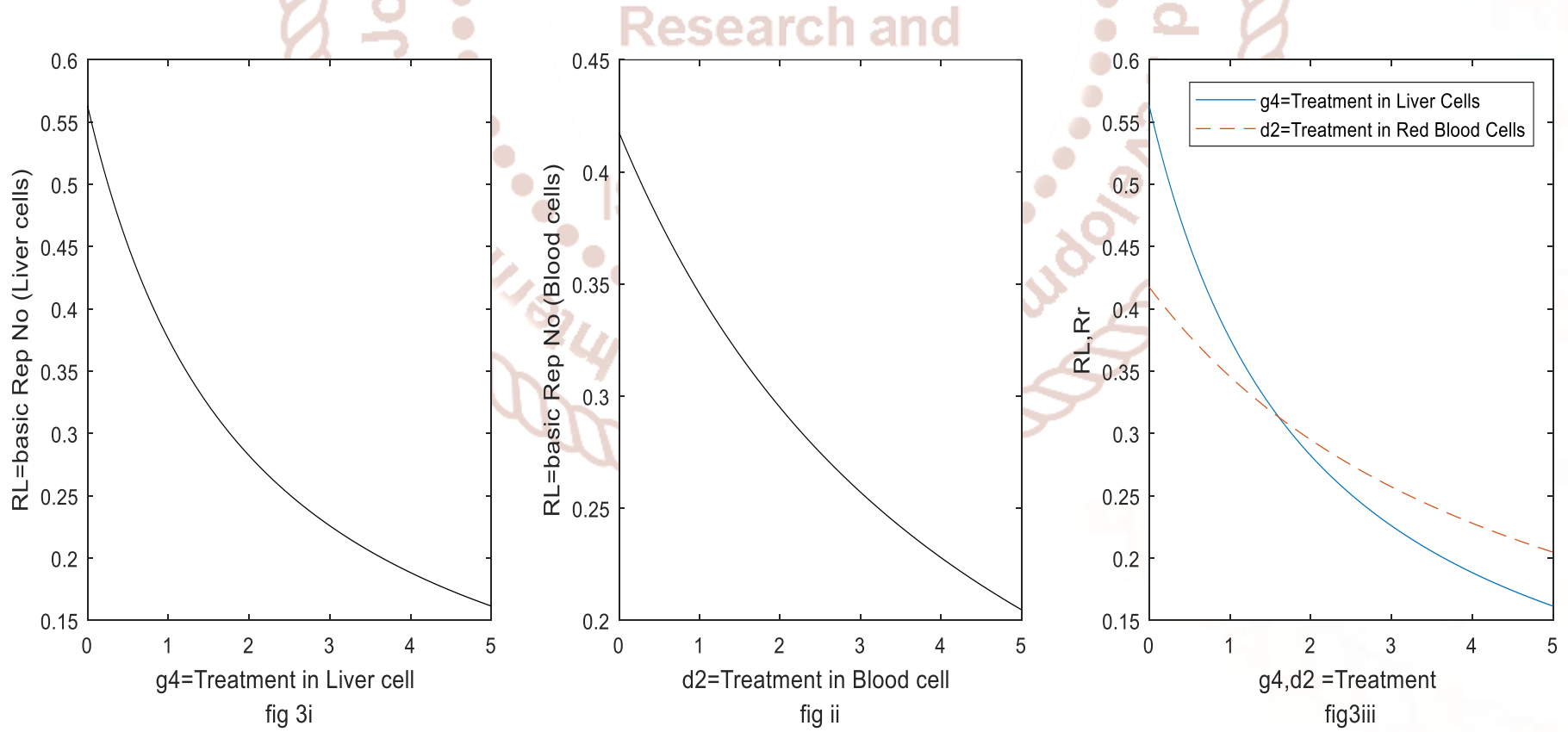

Figures (3i - 3iii): Numerical Simulation of the Basic Reproduction Number R_L and $r$ using different rate of g_4 and d_2 (Treatment Control) 
International Journal of Trend in Scientific Research and Development (IJTSRD) ISSN: 2456-6470
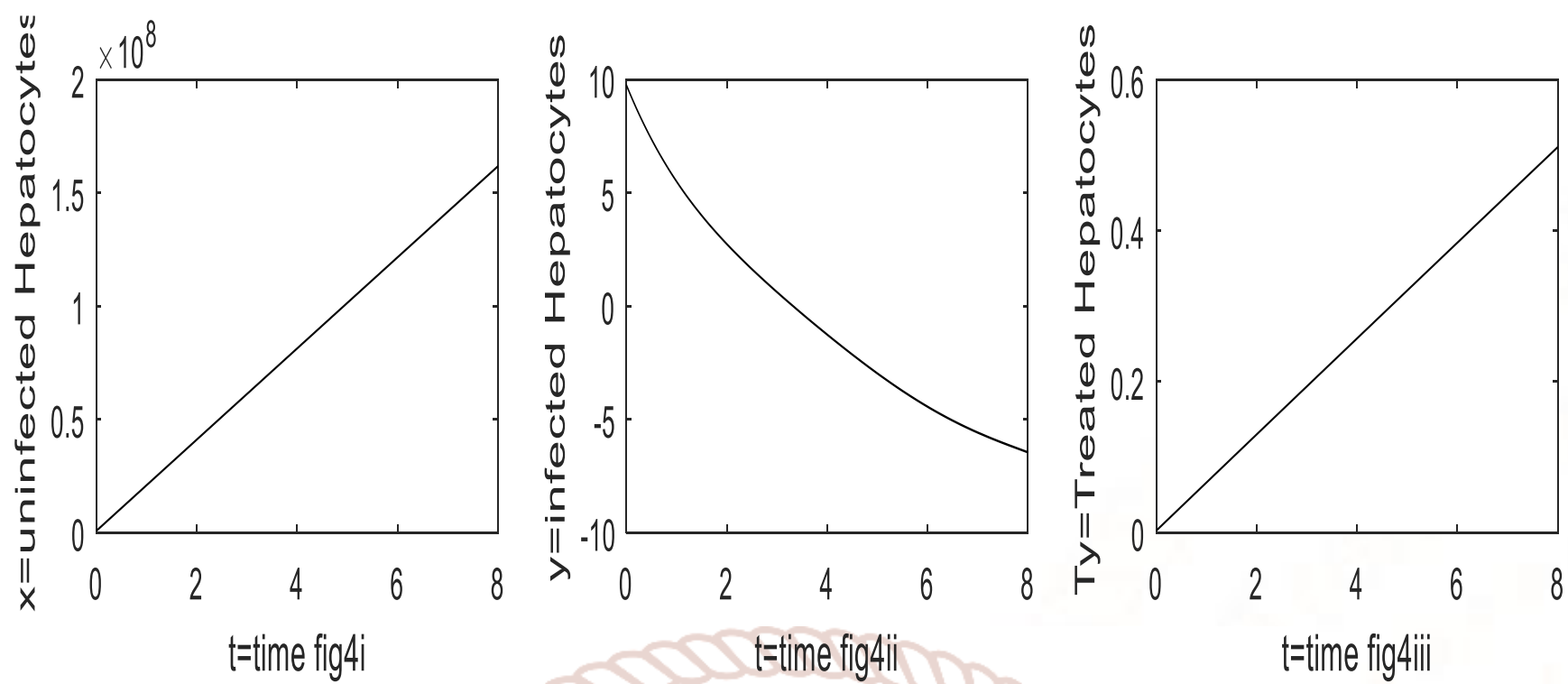
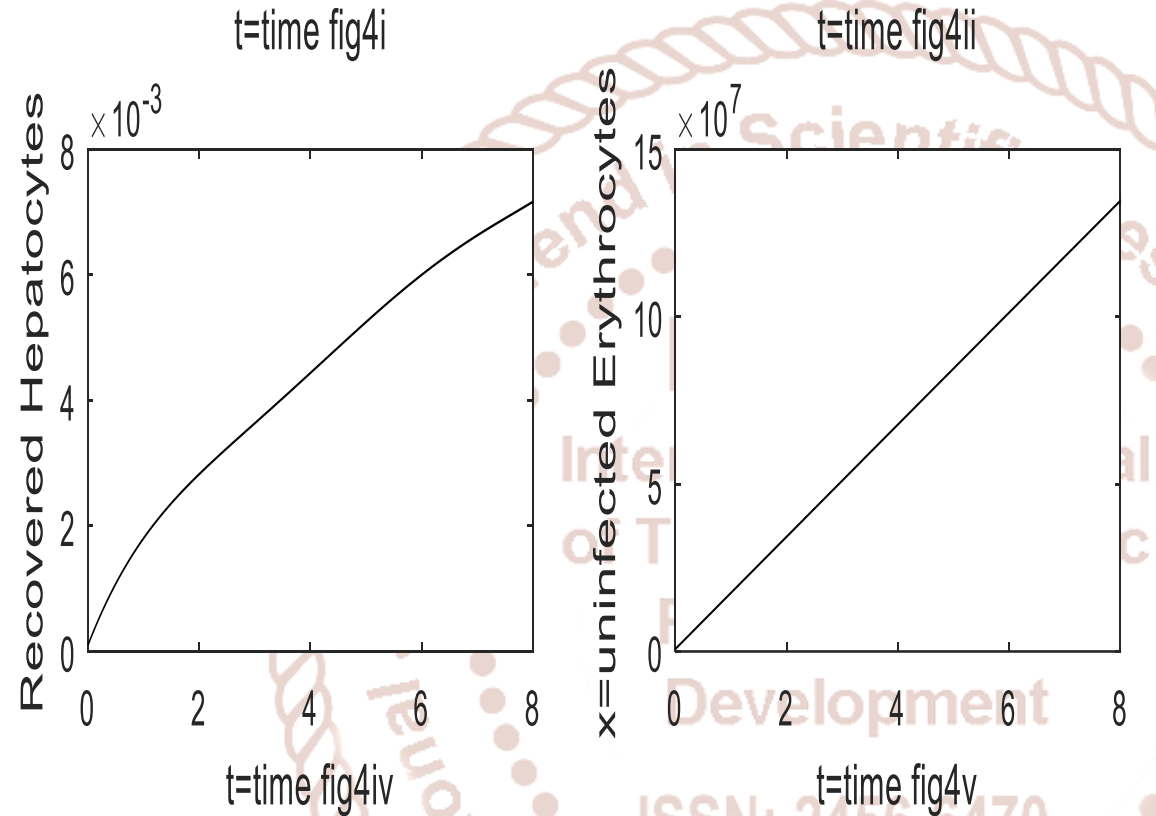

t=time fig $4 \mathrm{v}$
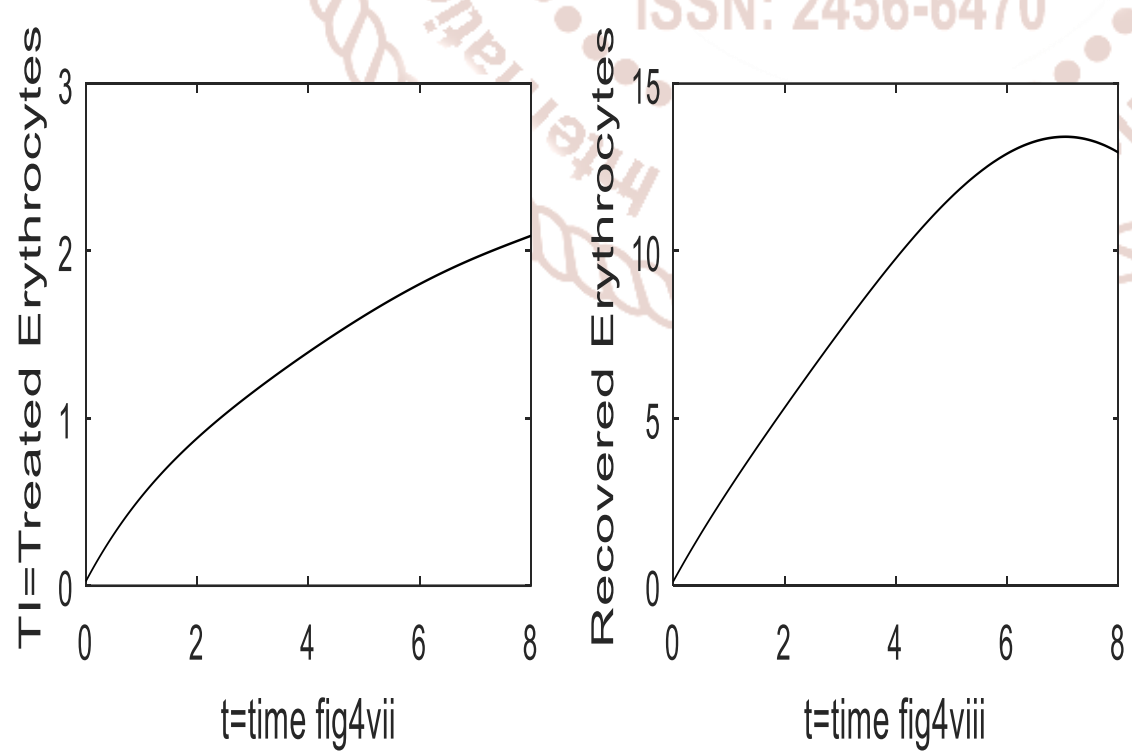

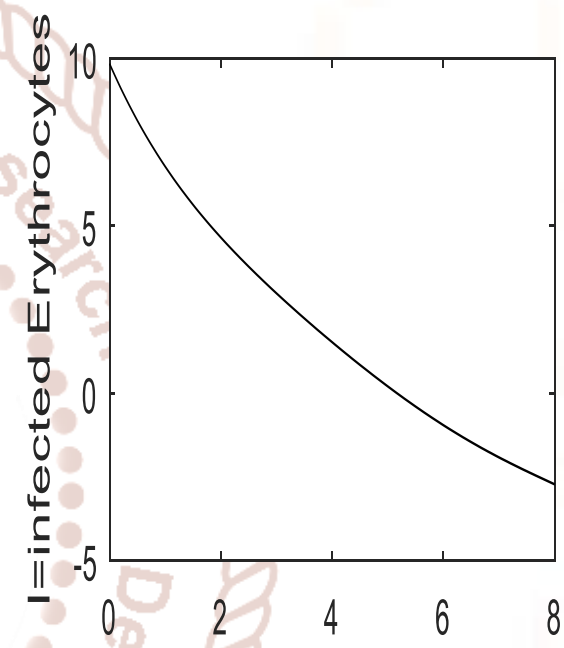

t=time fig4vi

Figures (4i - 4viii): Numerical Simulation of model system (3.3), when there are immune and treatment control from 0 -8days. 

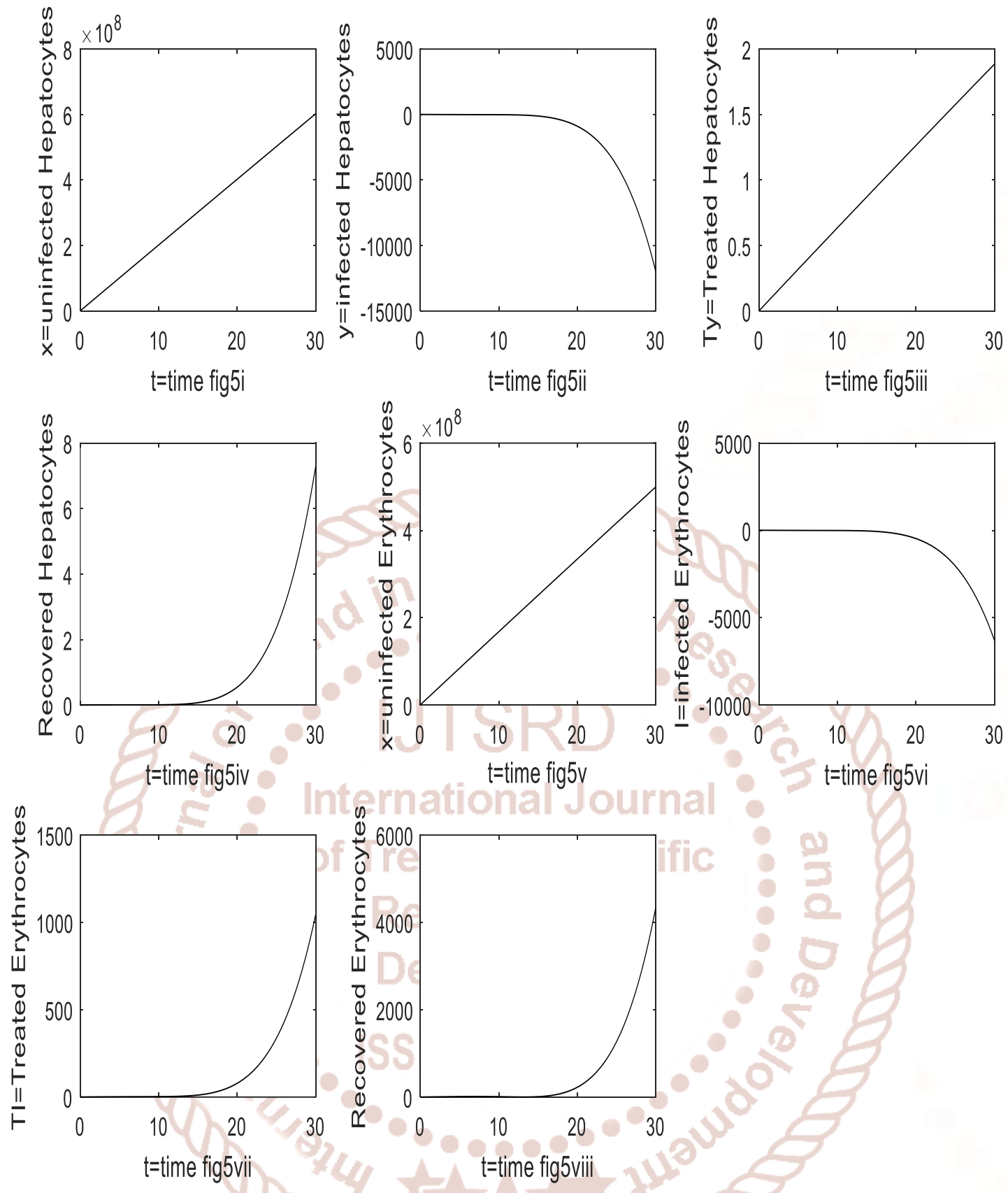

Figures (5i - 5viii): Numerical Simulation of model system (3.3), when there are immune and treatment control from 0 -30days.

The numerical simulation of immune response, treatment and disease free equilibrium point were performed to establish long term effects. Parameter values used in the simulations are given in table1. The simulation of the basic reproduction number, R_0 $=\mathrm{R} \_L+R \_r$ as in figures $2 \mathrm{i}-2 \mathrm{iii}$ shows that the immune response is effective in reducing the density of the parasites both in the liver cells and red blood cells. It indicates that the infection rate of the hepatocytes and erythrocytes are respectively reduced as the merozoites are suppressed and the sporozoites being cleared. Also, from figures $3 \mathrm{i}-3 \mathrm{iii}$, we observe that there is a perfect treatment since the reproduction numbers R_L and R_r under treatment are all less than one and $\mathrm{R} \_0=\mathrm{R} \_\mathrm{L}+\mathrm{R} \_\mathrm{r}$ is less than one. This implies that there exists the clearance of malaria parasites in both the liver and blood. Therefore with this reduction in infectious reservoirs, malaria can be greatly reduced in the population. If efficacy was equal to zero, that is $\mathrm{R} \_0=\left(\mathrm{R} \_\mathrm{L}+\mathrm{R} \_\mathrm{r}\right)>1$, immune response and treatment would have been useless.

Figures $4 \mathrm{i}-4 \mathrm{viii}$ and $5 \mathrm{i}-5 \mathrm{viii}$ show the disease free dynamics of malaria infection at hepatocytes of the liver and erythrocytes of the blood. The result shows that in the absence of malaria, the susceptible hepatocytes and erythrocytes respectively increases. Also, there is a sharp fall in the density of the 
infectious hepatocytes and erythrocytes. This indicates that the population of merozoites and sporozoites in the hepatocytes and erythrocytes will respectively decrease. Observe that new infectious mosquitoes repeatedly bite an individual to continue the life cycle to naïve individuals, activating the immune response against the infection. With an increase in treatment effectiveness, the density of the uninfected and recovered hepatocytes and erythrocytes increases, while the population of infected hepatocytes and erythrocytes decreases to lower value because the efficacy of the treatment used is high.

\section{Discussion and Conclusion}

The proposed study of the simulation of an intracellular differential equation model of the dynamics of malaria with immune control and treatment was designed and analyzed using ten compartments which were later simplified to eight compartments. The model studied malaria infection both in liver and blood. It also incorporated the effect of immune response and treatment of the infection respectively in the liver and blood stages. The analysis of the model as was presented by the positivity and existence of the systems solution shows that solutions exist. The results in this model indicate that the disease free equilibrium is asymptotically stable when $\mathrm{R} \_0=\left(\mathrm{R} \_\mathrm{L}+\mathrm{R} \_\mathrm{r}\right)<1$ and unstable when R_ $0=\left(R \_L+R \_r\right)>1$. in this study, the parameters, $\zeta, b \_3, b \_4$,and d_2 were significant in the successful clearance of malaria parasites. The sensitivity indices of these parameters were negative which indicates that increase in them results to reduction in malaria. The simulation result shows that with effective treatment, the density of uninfected hepatocytes and erythrocytes, treated hepatocytes and erythrocytes and recovered hepatocytes and erythrocytes increases. This simply means that the number of merozoites in the liver and sporozoites in the blood will be reduced and this implies clearance of malaria.

\section{Reference}

1. Adamu I. I. (2014). Mathematical model for the Dynamics and control of malaria in a population. Int. J. Pure Appl. Sci. Technol, , 20(1), pp1-12 .

2. Aly ASI, Vaughan A M and Kappe SHI. (2009). Malaria parasiteb development in mosquito and infection of the mammalian host. Annu. Rev Microbiol , 63: 195-221.
3. Andarson R M, May R M and Gupta S. (1989). Nonlinear phenomena in host - parasite interactions. Parasitology, 99:59-79.

4. Austin D J, White N J and Anderson R M. (1998). The dynamics of drug action on the within host population growth of infectious agents: Melding Pharmacokinetics with pathogen population dynamics,. J. Theoret. Biol, , 194: 313-339.

5. Bennink S, Kiesow M J and Pradel G. (2016). The development of malaria parasites in the mosquito midgut, . Cellular Microbiology, Published by John wiley and son Ltd, 18(7) 905-918.

6. Billker O, Shaw M K, Margos G and Sinden R E. (1997). The role of temperature, $\mathrm{pH}$ and mosquito factors as triggers of male and female gametogenesis of plasmodium bergher in vitro. Parasitology , 15:1-7.

7. Birkhoff G and Roff G C (1989) ordinary differential equation, John Wiley and sons, New York, NY, USA, USA $4^{\text {th }}$ edition.

8. Castillo-Requelme M, McIntyre D and Barnes K. (2008). Household burden of malaria in south Africa and Mozambique: is there a Catastrophic impact? Tropical Medicine and international health, 13(1) 108-22.

9. Chi-Johnston G L. (2012). Mathematical modeling of malaria: Theories of Malaria Elimination. Columbia University Academic Commons, https://doi.org/10.7916/D8Tx3NFM .

10. Chitnis N, Hyman J M and Cushing J M. (2008). Determining important parameters in the spread of malaria through the sensitivity analysis of a mathematical model. Bulletin of mathematical biology, 70(5), 1272-1296.

11. Chiyaka C, Garira W and Dube S. (2010). Using mathemathics to understand malaria infection during erythrocytic stage. Zimbabwe J. Sci. Technol, 5: 1-11.

12. Diekmann O and Heesterbeek J A P and Metz J A $J$ (1990). On the definition and conputation of basic reproduction number Ro in models for infectious diseases in heterogeneous populations. J. Math. Biol. , 28, 365-382.

13. Dondorp A M, Kager P A, Vreeken J and White NJ. (2000). Abnormal blood flow and red blood cell deformability in severe malaria parasitol. today, 16: 228-232. 
14. Ducrot A, Some B, Sirima S B and Zongo P. (2008). A mathematical model for malaria involving differential susceptibility, exposedness and infectivity of human host.

15. Esteva L, Gumel A B and De Leon C V. (2009). Qualitative study of transmission dynamics of drug - resistant malaria. Matrh. Comput. Modelling , 50: 611-630.

16. Garcia G E,Wirtiz R A, Barr J R, Woolfitt A and Rosenberg R. (1998). Xanthurenic acid induces gametogenesis in plasmodium, the malaria parasite. J Biol Chem , 273: 12003-12005.

17. Ghosh A K, Jacob-Lorena. M. (2009). Plasmodium Sporozoite invasion of the mosquito salavary gland. Curr Opin Microbiol, 12:394400.

18. Hommel M and Gilles H. M. (1998). Malaria in topley and wilson's microbiology and microbial infections. parasitology, ninth edition, vol.5. chapter 20.

19. Johansson P and Leander J. (2012). Mathematical modeling of malaria- methods for simulation of epidemics. A report from Chalmers University of Technology Gothenburg, .

20. Kar T K and Jana S. (2013). A theoretical study on mathematical modeling of an infectious Disease with application of optimal control. Biosystems, vol. 111.no. 1, pp.37-50.

21. Kawamoto F, Alejo-blanco R Fleck S L and Sinden RE. (1991). Plasmodium Bergher. Ionic regulation anf the induction of gametogenesis. Exp. Parasitol , 72:33-42.

22. Kuehn A and Pradel G. (2010). The coming-out of malaria gametocytes. . J. Biomed, 976827.

23. Malaguamera L and Musumeci S (2002). The immune response to plasmodium falcparum malaria. the Lancet Infection disease, Vol.2, pp $472-478$

24. Menard R, Tavares J Cockbum I, Markus M, Zavala F and Amino R . (2013). Looking under the skin: the first steps in malaria infection and immunity. Nat. Rev. Microbiol , 11:701-12.

25. Mohammed A O and Orukpe P. E. (2014). Modified mathematical model for malaria control. International Journal of Applied Biomedical Engineering vol.7, No. 1.
26. Molineaux L and Gramiccia G. (1980). The Garki Project, Research on the epidemiology and control of malaria in sudan savanna of west Africa. World Health Organzation, Geneva .

27. Mota M M, Pradel G, Vanderberg J P, Hafalla J C, Frevert U, Nussenzweig RS and Rodriguez A. (2001). Migratio of plasmodium sporozoitesthrough cells before infection. Science , 29: 141-144.

28. Olaniyi S and Obabiyi O S. (2013). mathematical Model for Malaria Transmission Dynamics on Human and Mosquitopopulation with nonlinear force of Infection. International Journal of Pure and Applied Mathematics , 88(1) : 125-150.

29. Pradel G. (2007). Proteins of the malaria parasite sexual stages: expression, Function, and potential for transmision blocking strategies. Parasitology , 134: 1911-29.

30. Shah N H and Gupta J. (2013). SEIR model and simulation for vector Born Diseases. . Applied mathematics , 4, 13-17.

31. Sologub L, Kuehn A, Kern S Przyborski J, Schillig R and Pradel G(2011). Malaria proteases mediate inside-out egress of gametocytes from red blood cells following parasite transmission to the mosquito. cell microbiol. 13: 897-912.

32. Tabo Z, Luboobi L S and Ssebukliba J (2017). Mathematical modelling of the in-host dynamics of malaria and the effects of treatment. J. Math. Computer Sci., 17:1-21.ZVan den Driessche P and Watmough J. (2002). Reproduction number and sub threshold endemic equilibria for compartmental models of disease transmission. Math. Biosci. , 180, 29-48.

33. World Health Organization. (2015). Global Report on anti malarial Drug Efficacy and Drug Resistance. Tech- Rep, World Health Organization.

34. World HealthOrganization. (2013). World Malaria Report. WHO Global Malaria Program, Geneva 2013.

35. Zhien M, Yieang Z and Jianhong W (2009). Modeling and dynamics of infectious disease. World scientific publishing Co-Pte Ltd. Singapore. 